\title{
ADAPTIVE CONTROL OF TIME-INVARIANT SYSTEMS WITH DISCRETE DELAYS SUBJECT TO MULTIESTIMATION
}

\author{
M. DE LA SEN AND S. ALONSO \\ Received 14 November 2005; Accepted 27 February 2006
}

This paper deals with a robustly stable adaptive pole-placement-based controller for timedelay linear systems with unknown point delays within known intervals of sufficiently small lengths under unmodeled dynamics and bounded disturbances. A multiestimation scheme is used to improve the identification error and then to deal with possible errors between the true basic delays compared to that used in the regressor of the adaptive scheme. Each estimation scheme possess a relative dead zone for each estimation scheme which freezes the adaptation for small sizes of the adaptation error compared with the estimated size of the contribution of the uncertainties to the filtered output. All the estimation schemes run in parallel but only that, which is currently in operation, parameterizes the adaptive controller to generate the plant input at each time. A supervisory scheme chooses in real time the appropriate estimator subject to a minimum residence time which is the tool to ensure closed-loop stability under switching between the estimators in the estimation scheme. The dead zone adaptation mechanism prevents the closed-loop system against potential instability caused by uncertainties.

Copyright (c) 2006 M. De la Sen and S. Alonso. This is an open access article distributed under the Creative Commons Attribution License, which permits unrestricted use, distribution, and reproduction in any medium, provided the original work is properly cited.

\section{Introduction}

Recent research in adaptive control has been devoted to robustness issues of continuous and discrete adaptive systems against unsuitable unmodeled dynamics and presence noise and to the relaxation of classical assumptions like the stability of the plant inverse and the knowledge of the sign of the high frequency gain (see, e.g., $[7,11,14,15,18,24]$ ). On the other hand, it is well known that time-delay systems are a natural way for modeling some real processes like population growth, signal and fluid transmission, war and peace models. Such systems have an infinite spectrum and the associate modes cannot be ensured to be close to their undelayed counterparts as the delay size increases what typically might cause instability $[2,8,22]$. Important work has been devoted to the stability and

Hindawi Publishing Corporation

Discrete Dynamics in Nature and Society

Volume 2006, Article ID 41973, Pages 1-27

DOI 10.1155/DDNS/2006/41973 
stabilization $[5,13,16,17,19,26]$ and robust stability and stabilization $[3,22,23,26]$ of such systems. The design of memoryless stabilizing controllers has been considered in $[5,17,26]$ while the design of delay-dependent controllers has been considered in $[16,19]$. In [5], the use of alternative stabilizing control laws with finite or infinite memory for systems subject to bounded point delays has been considered. The adaptive control problem for systems under internal point delays has also been considered (see, e.g., $[6,10]$ and references therein). The main drawbacks arise when internal delays are present since they typically cause an infinite asymptotic closed-loop spectra, like in the nonadaptive case, unless the adaptive controller compensates for the presence of plant delays. The point of view adopted in [10] has been to consider the choice of either a finite or infinite spectrum in the reference model due to design requirements. The first situation applies to the cases when the presence of delays is parasitic while the second one is useful for those when the presence of the plant delays is adequate for the closed-loop system. In those papers the delay was assumed to be exactly known.

Throughout this paper, the plant is assumed to be linear and possibly subject to unmodeled dynamics and bounded noise, and it possibly operates under slightly unknown commensurate point delays belonging to a given known real interval of small known length and location. It is not assumed to be inversely stable and an overbounding function of the contribution of the uncertainties dynamics to the output is not requested to be known. Furthermore, the plant parameter vector is unknown and assumed to belong to a known convex set for all time while its time derivative is not necessarily known and allowed to be impulsive at isolated points. Those potential impulses correspond to discontinuities in the parameter estimates due to switches in between the various estimation schemes. The main objective of the paper is to derive a robustly stable parameter-adaptive scheme for linear time-invariant systems under unknown constant point delays of small maximum allowable deviation with respect to a known given nominal value in the possible presence of unmodeled dynamics and bounded noise. A multiestimation scheme is used to improve the identification error and then to deal with possible errors between the true basic delay compared to that used in the regressor of the adaptive scheme. Each estimation scheme (or estimator) possesses a relative dead zone which freezes the adaptation for small sizes of the adaptation error compared to the size of the contribution of the uncertainties to the filtered output which is estimated via a known overbounding function of the contribution of the uncertainties to the output (see, e.g., $[7,14-16,19]$ for the delay-free case). All the estimation schemes run in parallel but only that which is currently in operation parameterizes the adaptive controller to generate the plant input at each time. This strategy guarantees that the parameter estimation is not disrupted by small identification errors. The adaptive controller synthesis is based on a pole-placement design for the case of nominal (i.e., disturbance-free) known delay-free plant.

One of the basic design tools used for adaptive stabilization in the presence of a wide class of unmodeled dynamics and bounded noise is the adaptation dead zone; see, for instance, $[7,14]$. Such dead zones are implemented in such a way that the estimates are maintained constant when the absolute value of the prediction error is small compared to the size of the contribution of the uncertainties to the output. In [7], the overbounding function of the contribution of the various uncertainties to the filtered output, 
which is needed to build the relative adaptation dead zone, is measurable while it has been estimated by extending the estimation scheme in [14]. An alternative technique to achieve robust stability has been the combination of standard estimation procedures with projections of the estimates on known convex sets within which the stabilization is guaranteed (see [7, 14, 15, 24] and related references for details). Also, additional effort has been devoted in the last years to alleviate some of the cumbersome assumptions usually made on the controlled plant in the classical formulation in adaptive control. In particular, a controllability condition was obtained in [25] by using switching between different tuned controllers while the use of excitation in near-singular cases was proposed in [12]. On the other hand, the plant is not assumed to be controllable for adaptive stabilization in [4]. In those papers, such a technique has been, in particular, applied to robust adaptive control of a class of nominally first-order hybrid systems. In such a class, the continuous-time output is simultaneously driven by the continuous-time input and preceding samples of the input and output at sampling instants. More recently, the use of a set of simultaneous estimators (multiestimation) has been proposed in order to improve the adaptive scheme's performance. The basic mechanism usually consists basically of switching at certain times the current controller to the one associated with a better registered performance. The performance evaluation is made according to a supervisory evaluation of performance in terms of an appropriate quality index being the weighted time-integral of the square tracking or tuning errors over some past time interval. See, for instance, $[20,21]$. A practical reason to proceed in that way is that the use of several simultaneous estimation schemes, perhaps subject to different initial conditions, allows easily dealing with possible changes in the plant operation points and with possible poor adaptation transients associated with a unique estimation scheme. A general multiestimation framework has been provided in [20] while each estimation operates for all time by generating a potential plant input but only one of them is injected to the system during appropriate time intervals from each controller switching to the next consecutive one. A related localization-based switching technique proposed in [27] for time-varying discrete systems ensures that the control switching converges rapidly. Multiestimation schemes for discrete systems with robust identification and control issues have also been recently proposed for discretization under zero-order, first-order, and fractional-order hold circuits, $[1,9]$.The closed-loop stability is preserved by appropriate selection of the switching times between controllers in all the above papers. The particular technique proposed $[20,21]$ to evaluate a loss performance of square integral with forgetting factor type of the identification error measured on some past time interval. The control strategy consists basically of switching to the current adaptive controller corresponding to some of the estimators in simultaneous operation to the one leading to the minimum cost according to such a function. That controller is maintained in operation until a new minimum cost is achieved. A predesigned minimum residence time prevents against possible infinitely fast switching and ensures the existence of the problem solution.

As proposed in $[20,21]$, all the estimation schemes operate simultaneously on the plant but the control input is generated as a convex linear combination of the set of potential control signals each associated with the individual adaptive controller associated with each estimator. The proposed multiestimation technique is suboptimal since all the 
required data are not known "a priori" when the bang-bang optimal strategy is applied for a performance index so as to calculate the weighting functions for evaluating the convex combination for either the controllers or the estimators. The plant is allowed to possess stable pole-zero cancellations which are not required to be known and do not influence the adaptive controller parameterization. In this way, the main properties of the adaptive scheme are independent of the plant physical realization being minimal or not and (if it is nonminimal) on the number, multiplicity, and location within the stability region of the unobservable and/or uncontrollable modes. The multiestimation philosophy could potentially work successfully when the estimation schemes have the same structures but, for instance, different initial conditions and/or different free-design parameters in the adaptive algorithm or when the estimators manipulate distinct structures of the updating algorithms. It has also been proved to be useful when the various estimators run over distinct parameter sets of the parameter space involving projections on the respective boundaries. Robust closed-loop stability is guaranteed for the class of uncertainties dealt with in $[7,14]$. It is also pointed out that the proposed technique can be combined with supervision ones over past measurements to calculate the estimator weights. The extension of the proposed technique to the use of any finite number of estimators while preserving the robust stability of the closed-loop system is also focused on.

In summary, the main novelties of the proposed scheme consist of relevant generalizations of previous work existing in the literature for delay-free systems mainly consisting of relaxing some hypotheses and design constraints. Those generalizations consist basically of the features that the delays are not exactly known, the system can possess known stable zero-pole cancellations, a multiestimation scheme is incorporated for point time-delay systems to improve the identification performance, and then the control performance, while unmodeled dynamics and bounded noise are allowed. The constants describing the over-bounding function are not necessarily known while they might be incorporated to the estimation scheme when unknown.

The paper is organized as follows. Section 2 is devoted to the problem statement with the plant and multiestimation models. Section 3 is firstly concerned with the adaptive controller synthesis for the case of known plants. The design relies on the solvability of a number of coupled polynomial diophantine equations directly related to the delayed plant structure. Then, the basic adaptive control scheme is presented together with the use of a set of parallel-type multiestimation schemes, which is especially useful for the case when the plant delays are not known precisely. The robust stability properties of the closed-loop system are also established and discussed. Section 4 presents a simulated example and, finally, conclusions end the paper. The mathematical proofs of the various results are given in Appendices A and B. The key technical feature is to prove the exponential stability of the delayed system provided that the delay-free one is exponentially stable of sufficiently large stability abscissa and the base delay error and delayed dynamics are sufficiently small.

Notation 1.1. (i) $D:=d / d t$ is the time-derivative operator formally equivalent to the Laplace operator $s$. Consequently, $D^{i+1}=D \cdot\left(D^{i}\right)=d^{i+1} / d t^{i+1}$ with $D^{0}=1$. Also, $e^{-h D}$ is the base time-delay operator for the base delay $h$, the commensurate internal delays being $h_{k}=k h, k=1,2, \ldots, q$. 
(ii) $\partial(\cdot)$ stands for the degree of the $(\cdot)$-polynomial and $\partial_{s}(\cdot)$ stands for the degree with respect to the variable $s$ of a quasipolynomial in $\left(s, e^{-h s}\right)$.

(iii) The notation $v(t)=G(s)\left[v_{0}\right]=g^{*} v_{0}$ is the zero-state response at time $t \geq 0$ of the realizable filter $G(s)=B\left(s, e^{-h s}\right) / A\left(s, e^{-h s}\right)$ for the signal input $v_{0}(\tau)$ for all $\tau \in[0, t]$, where $B\left(s, e^{-h s}\right)$ and $A\left(s, e^{-h s}\right)$ are quasipolynomials of degrees satisfying $\partial_{s} B \leq \partial_{s} A-1$, and $g(t)$ is the impulse response of $G(s)$, that is, the Laplace inverse transform of $G(s):=$ $\operatorname{Lap}(g(t))$, which is the Laplace transform of $g(t)$. The set of differential equations whose zero-state solution is $v(t)$ under input $u(t)$ is represented by $A\left(D, e^{-h D}\right) E\left(D, e^{-h D}\right) v(t)=$ $B\left(D, e^{-h D}\right) E\left(D, e^{-h D}\right) u(t)$.

(iv) Consider the differential equation $\bar{A}(D) v(t)=\bar{B}(D) u(t)+\eta(t)$ with $\bar{A}(D)=$ $A(D) E(D)$, and $\bar{B}\left(D, e^{-h D}\right)=B\left(D, e^{-h D}\right) E\left(D, e^{-h D}\right)$ denotes an uncertain linear and timeinvariant plant. Thus, the term "nominal plant" applies to the uncertainty-free (i.e., $\eta \equiv$ 0 ) plant modeled by $\bar{A}\left(D, e^{-h D}\right)=v_{0}(t)=\bar{B}\left(D, e^{-h D}\right) u(t)$. The name "nominal transfer function" applies to $G(s)=B\left(s, e^{-h s}\right) / A\left(s, e^{-h s}\right)$, that is, the (cancellation-free) perfectly modeled transfer function.

(v) The time argument, as well as the arguments $D$ and $s$, is sometimes omitted in the explicit notation for the sake of notation simplicity when no confusion is expected.

(vi) $\lambda_{\min }(\cdot), \lambda_{\max }(\cdot)$, and trace $(\cdot)$ denote, respectively, the minimum, maximum, and trace of the $(\cdot)$-matrix. In particular, $\beta_{1} \leq \lambda_{\min }(P(t))$ and $\beta_{2} \geq \lambda_{\max }(P(t))$ for $P(t)$ being the covariance matrix of the estimation algorithm.

(vii) The $l_{2}$-matrix norm is denoted by $\|(\cdot)\|_{2}$. If a subscript for norm is not used, it is meant that the kind of norm is irrelevant.

(viii) $L_{\infty}$ is the set of scalar or vector real functions $f: \mathbb{R}_{0}^{+} \rightarrow \mathbb{R}^{\mathbf{n}}$, some $n \geq 1$, such that $|f(t)|<\infty$ for all $t \in \mathbb{R}_{0}^{+}$with $\mathbb{R}_{0}^{+} \equiv \mathbb{R}^{+} \cup\{0\}=[0, \infty) \cap \mathbb{R}$.

(ix) $L_{p}$ is the set of scalar real functions $f: \mathbb{R}_{0}^{+} \rightarrow \mathbb{R}$ such that $\int_{0}^{\infty}|f(\tau)|^{p} d \tau<\infty(n \geq 1)$.

(x) Scalar and/or vector functions $f, g: \mathbb{R}_{0}^{+} \rightarrow \mathbb{R}^{\mathbf{n}}(n \geq 1)$ are $f=O[g]$ if $f(t) \leq K_{1} g(t)+$ $K_{2}$, some real constants $K_{1,2} \geq 0$, and $f=o[g]$ if $f=O[g]$ and $\lim _{t \rightarrow \infty}(f(t) / g(t))=0$.

\section{Plant structure and multiestimation scheme}

2.1. Plant. Consider the single-input single-output $n$ th-order continuous-time linear time-invariant system with $q$ internal point commensurate delays $h_{k}=k h(k=1,2, \ldots, q)$ :

$$
A\left(D, e^{-h D}\right) y(t)=B\left(D, e^{-h D}\right) u(t)+\eta(t),
$$

where $y(t), u(t)$, and $\eta(t)$ are the scalar output, input, and a signal that quantifies the contribution of the unmodeled dynamics and bounded disturbances to the output, respectively, with $D \equiv d / d t$ and $e^{-h D}$ being the time-derivative and time-delay operators, respectively. Those operators are explicitly defined as $\dot{v}(t)=d v(t) / d t=D v(t)$ and $v(t-$ $h)=e^{-h D} v(t)$ which satisfy the recursions $v^{(i)}(t)=d v^{(i)}(t) / d t^{(i)}=D v(t)=D v^{(i-1)}(t)$ and $v^{(i)}(t-j h)=e^{-j h D} v^{(i)}(t)=e^{-h D} v^{(i)}(t-(j-1) h)$, for $i=1,2, \ldots, n$ ( $n$ being the order of (2.1)), $j=1,2, \ldots, q$ ( $q$ being the number of internal delays), respectively, with $D^{0}=1$. Note that, since $D$ is formally analogous to the Laplace operator " $s$," the transfer function associated with (2.1), that is, the quotient of Laplace transforms of the output to 
the input, $Y(s) / U(s)$, under zero initial conditions is formally obtained from (2.1) by directly replacing the operators $D \rightarrow s$ and $e^{-h D} \rightarrow e^{-h s}$. Such a transfer function is a strictly proper (since $m<n$ ) rational function (i.e., meromorphic) of complex variable, obtained as a quotient of quasipolynomials, in the indeterminate " $s$." It is also depending explicitly on $e^{-h s}$ because of the presence of the delays. Note that the nominal plant is described by (2.1) when $\eta \equiv 0$. Any possible cancellation quasipolynomial $E\left(D, e^{-h D}\right)$ as referred to in the notation is strictly stable and it has been cancelled in (2.1) and included in $\eta(t)$ although this is not explicitly reflected in the notation. In this context, the formalism derived in this paper applies also directly to any stable nonminimal realization of $(2.1)$ described by $\bar{A}\left(D, e^{-h D}\right) y_{f}(t)=\bar{B}\left(D, e^{-h D}\right) u_{f}(t)+\bar{\eta}_{f}(t)$, where

$$
\bar{A}\left(D, e^{-h D}\right)=A\left(D, e^{-h D}\right) E\left(D, e^{-h D}\right), \quad \bar{B}\left(D, e^{-h D}\right)=B\left(D, e^{-h D}\right) E\left(D, e^{-h D}\right),
$$

and $\bar{\eta}_{f}(t)=E\left(D, e^{-h D}\right) \eta_{f}(t)$ with $\partial_{s} E \leq n$ provided that $E\left(D, e^{-h D}\right)$ has all its zeros in Res $<0$, and $\left(A_{0}(D), B_{0}(D)\right)$ is a controllable and observable pair, that is, cancellation-free (Assumption 2.1(2)). If $E\left(D, e^{-h D}\right) \neq 1$, then $E\left(D, e^{-h D}\right)$ gives extra poles to the reference model which are not cancelled with zeros and which have to be taken into account to establish its stability abscissa, that is, the maximum of the real parts of all its eigenvalues which is negative since the reference model is stable. That extension is direct and no related comments will be further given. The functions of initial conditions $\varphi:[-q h, 0] \rightarrow \mathbb{R}$ of $(2.1)$ are defined as $\varphi(t)=\varphi^{(1)}(t)+\varphi^{(2)}(t)$, where $\varphi^{(1)}:[-q h, 0] \rightarrow \mathbb{R}$ is a piecewise continuous real $n$-vector function, and $\varphi^{(2)}:[-q h, 0] \rightarrow \mathbb{R}$ is a function of bounded discontinuities on a subset of zero measure of $[-q h, 0]$; that is, it consists of a finite set of bounded discontinuities so that it is of support of zero measure. The initial conditions might be defined for direct input-output state-space realizations of (2.1) with the output $y(t)$ and its $(n-1)$ first derivatives as follows: $D^{j}(y(t))=\varphi_{j}(t), t \in[-q h, 0], j=0,1, \ldots, n-1$, with the simplified notation for $t=0, x_{j}(0)=\varphi_{j}(0)=x_{j 0}$ for $j=1,2, \ldots, n$. For each real $n$ vector function in the above class of initial conditions, there is a unique solution on $(0, \infty)$ for each piecewise continuous input from Cauchy-Peano existence theorem. $A\left(D, e^{-h D}\right)$ and $B\left(D, e^{-h D}\right)$ are quasipolynomials in the time-derivative and time-delay operators $D$ and $e^{-h D}$ defined by

$$
\begin{aligned}
& A\left(D, e^{-h D}\right)=\sum_{k=0}^{q} A_{k}(D) e^{-k h D}=\sum_{k=0}^{n} A_{k}^{\prime}\left(e^{-h D}\right) D^{k}=\sum_{k=0}^{q} \sum_{i=0}^{n} a_{k i} e^{-k h D} D^{i}, \\
& B\left(D, e^{-h D}\right)=\sum_{k=0}^{q} B_{k}(D) e^{-k h D}=\sum_{k=0}^{m} B_{k}^{\prime}\left(e^{-h D}\right) D^{k}=\sum_{k=0}^{q} \sum_{i=0}^{m} b_{k i}(D) e^{-k h D} D^{i},
\end{aligned}
$$

where $A_{(\cdot)}$ and $B_{(\cdot)}$ are polynomials defined as follows:

$$
A_{k}(D)=\sum_{\ell=0}^{n} a_{k \ell} D^{n-\ell} ; \quad B_{k}(D)=\sum_{\ell=0}^{m} b_{k \ell} D^{m-\ell} \quad(k=0,1, \ldots, q)
$$

with $a_{k \ell}, b_{j i}, k=0,1, \ldots, q, l=0,1, \ldots, n$, and $j, i=0,1, \ldots, m$ being real coefficients with the constraints $a_{00}=1$; that is, $A_{0}(D)$ is a monic polynomial, $b_{00} \neq 0$, and $m \leq n-1$. 
The two polynomials $A_{k}^{\prime}\left(e^{-h D}\right), k=0,1, \ldots, n$, and $B_{k}^{\prime}\left(e^{-h D}\right), k=0,1, \ldots, m$, are defined mutatis mutandis. The usefulness of the proposed notation for quasipolynomials in the operators $D$ and $e^{-h D}$ becomes obvious from the above four equations since the quasipolynomials are defined by real coefficients in two mutually dependent operator indeterminates $D$ and $e^{-h D}$ and, equivalently, by polynomial coefficients in each of the two indeterminates if the other indeterminate is the relevant variable in the description. Note that although the operator $e^{-h D}$ is dependent on the operator $D$, their jointly presence in the system dynamics makes that system to be infinite dimensional; that is, it possesses infinitely many modes since the denominator quasipolynomial of the associate transfer function possesses infinitely many zeros since it is a transcendent function. Note also that the combined use of the time-derivative and time-delay operators is easy to deal with. For instance, $D^{k} e^{-i h D} v(t)=v^{(k)}(t-i D)$ for any $k$ th time-differentiable signal $v(t)$. In order to improve the filtering properties to possible disturbances and to accommodate the adaptation transient rates in the adaptive case, define filtered signals from (2.1):

$$
y_{f}(t)=\frac{1}{F(D)} y(t) ; \quad u_{f}(t)=\frac{1}{F(D)} u(t) ; \quad \eta_{f}(t)=\frac{1}{F(D)} \eta(t)
$$

where $F(D)=D^{n}+\sum_{i=1}^{n-1} f_{i} D^{n-i}$ is an $n$ th-order monic Hurwitz polynomial of real constant coefficients. Thus, the filtered plant equation (2.1) becomes

$$
A\left(D, e^{-h D}\right) y_{f}(t)=B\left(D, e^{-h D}\right) u_{f}(t)+\eta_{f}(t)+v(t)
$$

so that the plant equation can be equivalently rewritten in regression form as

$$
\begin{aligned}
y(t) & =F(D) y_{f}(t)=\left(F(D)-A\left(D, e^{-h D}\right)\right) y_{f}(t)+B\left(D, e^{-h D}\right) u_{f}(t)+\eta_{f}(t)+v(t) \\
& =\theta^{T} \varphi(t)+\eta_{f}(t)+v(t),
\end{aligned}
$$

where $v^{(t)}$ is an exponentially vanishing signal associated with the initial conditions of the filters, and

$$
\begin{aligned}
& \theta^{T}=\left(\begin{array}{ccccccc}
\theta_{0}^{T} & \vdots & \theta_{1}^{T} & \vdots & \cdots & \vdots & \theta_{q}^{T}
\end{array}\right)
\end{aligned}
$$

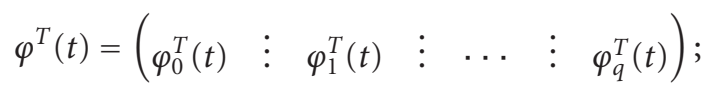

$$
\begin{aligned}
& \theta_{0}^{T}=\left(0, f_{1}-a_{01}, \ldots, f_{n-1}-a_{0, n-1} \quad \vdots \quad b_{00}, b_{01}, \ldots, b_{0 m}\right) ; \\
& \varphi_{0}^{T}(t)=\left(D^{n} y_{f}(t), D^{n-1} y_{f}(t), \ldots, y_{f}(t) \quad \vdots \quad D^{m} u_{f}(t), D^{m-1} u_{f}(t), \ldots, u_{f}(t)\right) ; \\
& \theta_{i}^{T}=\left(a_{0 i}, a_{1 i}, \ldots, a_{n i}: b_{0 i}, b_{1 i}, \ldots, b_{m i}\right) ; \\
& \varphi_{i}^{T}=\left(D^{n-1} y_{f}(t-i h), \ldots, y_{f}(t-i h) \quad \vdots \quad D^{m} u_{f}(t-i h), \ldots, u_{f}(t-i h)\right) \quad(i=1,2, \ldots, p) .
\end{aligned}
$$

The following assumptions are made on the plant (2.1). 
Assumption 2.1. (1) There exists a known bounded set $\Omega$ which is either a convex compact region or a connected union of a finite number of (disjoint or not) compact sets; that is, $\Omega=\bigcup_{i=1}^{p} \Omega_{i}$ such that $\theta \in \Omega$, some $i \in\{1,2, \ldots, p\}$.

(2) For all $\theta \in \Omega$, the corresponding polynomials $A_{0}(D)$ and $B_{0}(D)$ are relatively prime; that is, they have no common zeros when considered as complex functions of $D$ so that $\left(A_{0}(D), B_{0}(D)\right)$ is a controllable and observable pair.

(3) The base delay $h \in\left[h^{*}-\Delta h, h^{*}+\Delta h\right]$ may be unknown, where the nominal $h^{*}$ and the maximum error $\pm \Delta h(\Delta h \bullet 0)$ are both known.

(4) $\delta=\sum_{i=1}^{q}\left(\left\|A_{i}(D)\right\|+\left\|B_{i}(D)\right\|\right)$ is sufficiently small for all $\theta \in \Omega$.

Assumption 2.2. There exists a nonnegative function of time $\gamma: \mathbb{R}_{0}^{+} \rightarrow \mathbb{R}_{0}^{+}$satisfying $\gamma(t) \leq$ $\varepsilon_{1} \sup _{0 \leq \tau \leq t}\left(e^{-2 \rho 0(t-\tau)}\|\varphi(\tau)\|^{2}\right)+\varepsilon_{2}$ for some known real constant $\rho_{0}>0$ and possibly unknown real constants $\varepsilon_{1} \geq 0$ and $\varepsilon_{2} \geq 0$ such that $\left|\eta_{f}(t)\right|^{2} \gamma(t)$ for all time.

Remarks 2.3. Assumptions 2.1(1) and (2) are standard in pole-placement indirect adaptive control algorithms of time-invariant plants and lead to solvability of the diophantine equation associated with the synthesis of the pole-placement-based controller in the delay-free nominal case. It means that any delay-free plant (2.1) parameterized in $\Omega$ as well as its associate estimation model are both controllable and observable. Such a requirement can be easily relaxed and extended to the case when it is stabilizabled and detectable since the neglected stable cancellations cause an exponentially decaying neglected term in the control signal that does not modify the properties of the adaptive scheme. The controllability of the estimation model may be guaranteed for all time by using projection of the estimates on the boundary of the $\Omega$ domain, if necessary see, for instance, $[7,14]$. Note that, in particular, $\Omega$ may be a connected finite union of a finite number of bounded convex (disjoint or not) subsets that may be specifically relevant for the implementation of a parallel multiestimation scheme by specifying the cases when the estimates belong to some of those subsets or to the intersection of some subgroup of such subsets.

The projection technique is then incorporated in the estimation algorithm proposed in this paper. However, note that it is not required that neither the zeros of $B\left(D, e^{-h D}\right)$ nor those associated with the corresponding part of the estimation scheme are stable. Another alternative way which could be used to ensure the controllability of the estimated model for all time is the use of estimates modification procedures when the controllability of the estimated model is lost. The previously proposed modification procedures lead, in general, to high computational costs for delay-free plants of second order or higher (see, e.g., [7, 14] and references therein) that may lead to implementation difficulties. Assumptions 2.1(3) and (4) are used to guarantee closed-loop stability in the presence of uncertainties through the synthesis of a pole-placement-based adaptive controller even if the delays are unknown subject to Assumption 2.1(3). In addition, a global exponential adaptive closed-loop stability is also guaranteed in the absence of disturbances if the delay is unknown while belonging to a prescribed interval of sufficiently small measure (Assumption 2.1(3)). Finally, Assumption 2.2 holds if the signal $\eta_{f}(t)$ of the unmodeled dynamics contribution is the sum of a bounded disturbance and a signal related to the input by a strictly proper exponentially stable function. The constants $\varepsilon_{1}$ and $\varepsilon_{2}$ are not 
assumed known but estimated by extending the estimation scheme as proposed in [14] for the delay-free case.

2.2. Multiestimation algorithm. If the true parameter vector $\theta$ is unknown and replaced with any estimate $\hat{\theta}_{i}(t)$ of any of the estimation algorithms running in parallel $(i=1,2, \ldots, p)$, then the identification (or adaptation) error becomes

$$
\begin{aligned}
e_{i}(t)= & y(t)-\hat{y}_{i}(t)=y(t)-\hat{\theta}_{i}^{T}(t) \varphi(t)=\left(\widehat{A}_{i}\left(D, e^{-h D}, t\right)-A\left(D, e^{-h D}\right)\right) y_{f}(t) \\
& -\left(\widehat{B}_{i}\left(D, e^{-h D}, t\right)-B\left(D, e^{-h D}\right)\right) u_{f}(t)+\eta_{f}(t)+v(t) \\
= & -\tilde{\theta}_{i}^{T}(t) \varphi(t)+\eta_{f}(t)+v(t), \quad i=1,2, \ldots, p,
\end{aligned}
$$

where $\hat{y}_{i}(t)=\left(F(D)-\hat{A}_{i}\left(D, e^{-h D}, t\right)\right) y_{f}(t)+\widehat{B}_{i}\left(D, e^{-h D}, t\right) u_{f}(t)$ and $\tilde{\theta}_{i}(t)=\tilde{\theta}_{i}(t)-\theta$ are the output estimate and the parametrical error, respectively. $\widehat{A}_{i}\left(D, e^{-h D}, t\right)$ and $\widehat{B}_{i}\left(D, e^{-h D}\right.$, $t)$ are the estimates of $A\left(D, e^{-h D}\right)$ and $B\left(D, e^{-h D}\right)$, respectively, by the ith estimator $(i=$ $1,2, \ldots, p)$ with respective associate parametrical errors:

$$
\begin{aligned}
& \widetilde{A}_{i}\left(D, e^{-h D}, t\right)=\widehat{A}_{i}\left(D, e^{-h D}, t\right)-A\left(D, e^{-h D}\right) ; \\
& \widetilde{B}_{i}\left(D, e^{-h D}, t\right)=\widehat{B}_{i}\left(D, e^{-h D}, t\right)-A\left(D, e^{-h D}\right) .
\end{aligned}
$$

Assumption 2.4. $\delta_{j}(t)=\sum_{i=1}^{q}\left(\left\|\hat{A}_{j i}(D, t)\right\|+\left\|\hat{B}_{j i}(D, t)\right\|\right)$ is sufficiently small for all $\theta \in \Omega_{j}$, all $j=1,2, \ldots, p$, the $j$ th subscript denoting each estimator in the parallel scheme and all $t \bullet 0$.

The following least square-type multiple estimation algorithm is proposed. It involves an adaptation relative dead zone which is implemented for closed-loop stabilization under uncertainties and unknown base delay $h$ (under Assumption 2.2) is used for robust parameter estimation:

$$
\begin{gathered}
\dot{\hat{\theta}}_{i}(t)=P_{i}^{1 / 2}(t) \operatorname{Proj}_{i}\left\{b_{i}(t) P_{i}^{T / 2}(t) \varphi(t) e_{i}(t)\right\} ; \\
\dot{P}_{i}(t)=-b_{i}(t) P_{i}(t) \varphi(t) \varphi^{T}(t) P_{i}(t) ; \quad P_{i}(0)=P_{i}^{T}(0) \geq k_{0} I, \quad k_{0}>0,
\end{gathered}
$$

for $i=1,2, \ldots, p$, with $\operatorname{Proj}\{\cdot\}$ being a projection operator $[7,14,15]$ used to constraint the estimates of the $i$ th estimator within the bounded convex region $\Omega_{i}(i=1,2, \ldots, p)$ in the light of Assumption 2.1(1), and the relative adaptation dead zone being:

$$
\begin{gathered}
b_{i}(t)=\frac{\alpha_{1} s_{i}(t)}{1+\varphi^{T}(t) P_{i}(t) \varphi(t)} \\
s_{i}(t)= \begin{cases}0 & \text { if }\left|e_{i}(t)\right| \leq \vartheta \hat{\gamma}_{i}(t)^{1 / 2}, \\
1-\vartheta \frac{\hat{\gamma}_{i}(t)^{1 / 2}}{\left|e_{i}(t)\right|} & \text { otherwise },\end{cases}
\end{gathered}
$$


for $i=1,2, \ldots, p$, where $\vartheta>1$ is a design constant, and

$$
\begin{gathered}
\hat{\gamma}_{i}(t)=\left(\widehat{\varepsilon}_{i 1}(t), \widehat{\varepsilon}_{i 2}(t)\right)\left(\operatorname{Sup}_{0 \leq \tau \leq t}\left(e^{-2 \rho_{0}(t-\tau)}\|\varphi(\tau)\|^{2}, 1\right)\right)^{T} ; \\
\dot{\hat{\varepsilon}}_{i 1}(t)=\frac{\alpha_{1} s_{i}(t)}{2\left(1+\varphi^{T}(t) P_{i}(t) \varphi(t)\right)} \operatorname{Sup}_{0 \leq \tau \leq t}\left(e^{-2 \rho_{0}(t-\tau)}\|\varphi(\tau)\|^{2}\right) ; \\
\dot{\hat{\varepsilon}}_{i 2}(t)=\frac{\alpha_{1} s_{i}(t)}{2\left(1+\varphi^{T}(t) P_{i}(t) \varphi(t)\right)}
\end{gathered}
$$

with $\widehat{\varepsilon}_{i 1}(0)=\widehat{\varepsilon}_{i 2}(0)=0$ for any design constant $\alpha_{0}>0$, all $i=1,2, \ldots, p$. The last two equations estimate the constants defining the overbounding function of the disturbances (i.e., unmodeled dynamics and bounded noise). In the following, the parametrical error is defined as $\tilde{\theta}_{i}(t)=\hat{\theta}_{i}(t)-\theta$ and the errors for the constants of Assumption 2.2 are defined as $\tilde{\varepsilon}_{i j}(t)=\hat{\varepsilon}_{i j}(t)-\bar{\varepsilon}_{j}, i=1,2, \ldots, p, j=1,2$ for all $t \geq 0$.

2.3. Properties of the estimation algorithm. Note that the estimations of the $\varepsilon$-constants are positive and nondecreasing with time until a limit ensured by Theorem 2.5 is reached. Such a result, proved in Appendix A, is related to the properties of the multiestimation algorithm (2.9)-(2.13) irrespective of the control law provided that Assumptions 2.1-2.4 hold.

Theorem 2.5. The subsequent two items hold:

(i)

$$
\begin{gathered}
\left\|\tilde{\theta}_{i}\right\| \in L_{\infty}, \quad\left\|\hat{\theta}_{i}\right\| \in L_{\infty}, \quad\left|\tilde{\varepsilon}_{i} j\right|=\left|\hat{\varepsilon}_{i j}-\varepsilon_{i j}\right| \in L_{\infty}, \quad\left|\hat{\varepsilon}_{i j}\right| \in L_{\infty}, \\
\hat{\varepsilon}_{i j}(t) \longrightarrow \varepsilon_{i j \infty} \in(0, \infty) \quad \text { as } t \longrightarrow \infty, \text { for } j=1,2, i=1,2, \ldots, p ; \\
b_{i}\left(\hat{\gamma}_{i}-e_{i}^{2}\right) \in L_{\infty}, \quad\left(b_{i} \hat{\gamma}_{i}\right)^{1 / 2} \in L_{\infty}, \quad b_{i}{ }^{1 / 2}\left|e_{i}\right| \in L_{\infty}, \quad i=1,2, \ldots p \\
b_{i}\left(\eta_{f}-e_{i}\right)^{2} \in L_{\infty} \quad \text { if } \vartheta>1, \quad \| \dot{\hat{\theta}}_{i}|| \in L_{2} \cap L_{\infty}, \quad i=1,2, \ldots, p
\end{gathered}
$$

(ii)

$$
\begin{gathered}
b_{i}\left(\hat{\gamma}_{i}-e_{i}^{2}\right) \in L_{1} \cap L_{\infty}, \quad b_{i}^{1 / 2}\left(\hat{\gamma}_{i}^{1 / 2}+\left|e_{i}\right|\right) \in L_{1} \cap L_{\infty}, \\
b_{i}^{1 / 2}\left(\hat{\gamma}_{i}^{1 / 2}-\left|e_{i}\right|\right) \in L_{1} \cap L_{\infty},\left(b_{i} \hat{\gamma}_{i}\right)^{1 / 2} \in L_{1} \cap L_{\infty}, \quad b_{i}^{1 / 2}\left|e_{i}\right| \in L_{1} \cap L_{\infty} \quad \forall i=1,2, \ldots, p ;
\end{gathered}
$$

and all those signals tend asymptotically to zero as $t \rightarrow \infty$. Furthermore, $\left\|\dot{\tilde{\theta}}_{i}\right\| \in L_{2} \cap L_{\infty}$, $\hat{\theta}_{i}(t) \rightarrow \hat{\theta}_{i \infty}\left(\left\|\hat{\theta}_{i \infty}\right\|<\infty\right)$ as $t \rightarrow \infty$. Also, $b_{i}\left(\eta_{f}-e_{i}\right) \in L_{\infty}$, and $b_{i}\left(\eta_{f}-e_{i}\right)^{2} \rightarrow \infty$ as $t \rightarrow \infty$ if $\vartheta>1, i=1,2, \ldots, p$. 


\section{Adaptive controller design and closed-loop stability properties}

3.1. Adaptive control law. Each $i$-estimator, $i=1,2, \ldots, p$, generates a filtered control law candidate for each time $t$ :

$$
u_{i f}(t)=\frac{S_{i}\left(d, e^{-h D}, t\right)}{R_{i}\left(D, e^{-h D}, t\right)}\left(y_{f}^{*}(t)-y_{f}(t)\right), \quad i=1,2, \ldots, p,
$$

the filtered control law for time $\left[t_{i}, t_{i+1}\right)$ being

$$
u_{f}(t)=u_{i f}(t) \quad \text { some } i \in\{1,2, \ldots, p\}
$$

for all $t_{i} \in S$ (the sequence of switching times), where $S_{i}\left(D, e^{-h D}, t\right)$ and $R_{i}\left(D, e^{-h D}, t\right)=$ $R^{0}\left(D, e^{-h D}, t\right)+\widetilde{R}_{i}\left(D, e^{-h D}, t\right)$ are defined by time-varying quasipolynomials:

$$
S_{i}\left(D, e^{-h D}, t\right)=\sum_{k=0}^{q} S_{i k}(D, t) e^{-k h D}, \quad R_{i}^{0}\left(D, e^{-h D}, t\right)=\sum_{k=0}^{q} R_{i k}(D, t) e^{-k h D}
$$

defined through polynomials

$$
S_{i k}(D, t)=\sum_{\ell=0}^{m} s_{k \ell}^{(i)}(t) D^{\ell}, \quad R_{i k}(D, t)=\sum_{\ell=0}^{n} r_{k \ell}^{(i)}(t) D^{\ell}
$$

$(i=1,2, \ldots, p, k=0,1, \ldots, q)$, and a rational (in general transcendent) time-valued complex variable function

$$
\widetilde{R}_{i}\left(D, e^{-h D}, t\right)=-\frac{\widetilde{A}_{m}\left(D, e^{-h D}\right)}{\widehat{A}_{i}\left(D, e^{-h D}, t\right)},
$$

where the pair $\left(R_{k}(D, t), S_{k}(D, t)\right)$ satisfies uniquely the set of $(n+1)$ diophantine equations of time-varying polynomials:

$$
\begin{aligned}
\widehat{A}_{i 0}(D, t) R_{i k}(D, t) & +\widehat{B}_{0}(D, t) S_{k}(D, t) \\
= & A_{m k}(D)-\sum_{\ell=1}^{k}\left(\hat{A}_{i \ell}(D, t) R_{i, k-\ell}(D, t)-\widehat{B}_{i \ell}(D, t) S_{i, k-\ell}(D, t)\right)
\end{aligned}
$$

with $\partial\left(S_{i k}\right)=\partial\left(R_{i k}\right)-1=n-1$ for $k=0,1, \ldots, n$, provided that $\partial A_{m k} \leq 2 n(k=0,1, \ldots$, $m+1)$, since $\left(\widehat{A}_{i_{0}}, \hat{B}_{i 0}\right)$ are all coprime pairs $(i=1,2, \ldots, p)$ from the estimation projection and Assumption 2.1(1), and

$$
\tilde{A}_{i m}\left(D, e^{-h D}, t\right)=\sum_{k=m+1}^{2 q}\left[\sum_{\ell=\operatorname{Max}(0, k-n)}^{n} \hat{A}_{i \ell}(D, t) R_{i, k-\ell}(D, t)\right] e^{-k h D} .
$$

3.2. Model multiestimation and control switching rule. The choice of the current filtered control input (3.2a) from those ones generated by the overall parallel multiestimation scheme is made by the subsequent switching rule. Define $S=\left\{\ldots t_{i} ; i \geq 1\right\}$ as the 
finite (or infinite) set of switching instants between estimation models in $P=\{1,2, \ldots, p\}$ which satisfy the following. Let $t_{i} \in S$. Then, $t_{i+1} \in S$ if

(1) $T_{i}=t_{i+1}-t_{i} \geq T$ ( $T$ being the so-called minimum residence time) for all time in the switching sequence $S$;

(2) $j\left(t_{i+1}\right)=\operatorname{Min}\left(\ell \in p: j_{k}\left(t_{i+1}\right)\right.$, for all $\left.k \in p\right)$ such that $J\left(t_{i}\right)=J_{k 1}\left(t_{i}\right) \Rightarrow J\left(t_{i+1}\right)=$ $J_{k 2}\left(t_{i+1}\right)$ with $k_{1} \neq k_{2}$ for any estimators $k_{1,2} \in P$,

where $J_{i}(t)=\int_{t-T_{p}}^{t} e^{-\varsigma(t-\tau)}\left(\lambda e_{i}^{2}(t)+(1-\lambda) u_{i f}^{2}(t)\right) d \tau$, all $i \in P$ for some prescribed forgetting factor $\lambda>0$ and weighting factor $\lambda \in(0,1]$, which is a loss function which is a measure of a combined quality index for the identification and control effort. Note that each estimator is running for all time. However, the adaptive controller is parameterized by each estimator scheme during a time interval, subject to the above minimum residence time, before potential switching for reparameterization.

3.3. Main robust stability result. The combined equations (2.9) and (3.1) may be described through the auxiliary extended system

$$
\dot{x}(t)=\sum_{j=0}^{q} \mathbf{A}_{j}(t) x(t-j h)+b e(t)+g(t)+v(t),
$$

where the state vector, forcing signals, and parameterization are defined via the filtered input and output signals $u_{f}(t)$ and $y_{f}(t)$ together with their relevant time-derivatives up to order $n$ obtained from (2.5) as follows:

$$
\begin{aligned}
& x^{T}(t)=\left(D^{n-1} y_{f}(t), \ldots, D y_{f}(t), y_{f}(t) \quad \vdots \quad D^{n-1} u_{f}(t), \ldots, D u_{f}(t), u_{f}(t)\right)
\end{aligned}
$$

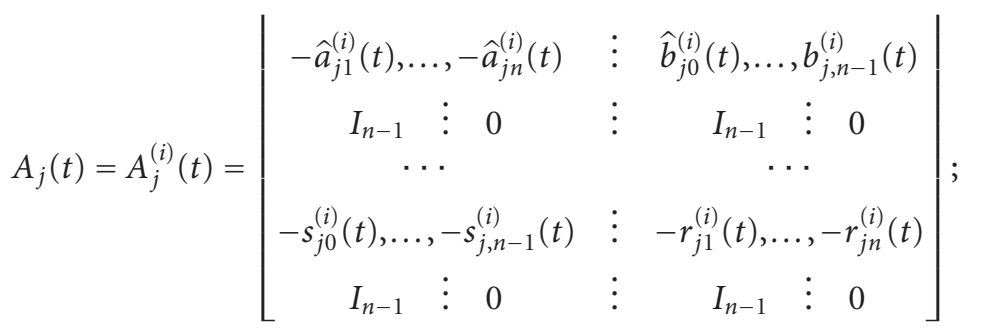

$$
\begin{aligned}
& G_{i}(t)=\left\lfloor\begin{array}{c}
0^{T} \\
s_{i, n-1}(t), \ldots, s_{i 0}(t) \\
0^{T}
\end{array}\right\rfloor \quad b_{T}=(1,0, \ldots, 0) ; \\
& g^{T}(t)=\sum_{j=0}^{n-1} G_{j}(t) y_{e f}^{*}(t-j h) ; \\
& y_{e f}^{* T}(t)=\left(D^{n-1} y_{f}^{*}(t), \ldots, D y_{f}^{*}(t), y_{f}^{*}(t)\right)
\end{aligned}
$$

for some $i$ th estimator $i \in\{1,2, \ldots, p\}$, all $j=1,2, \ldots, q$, running and generating the plant 
control input at any time $t$. The vector signal $v(t)$ is generated by the initial conditions of the filters. It may be directly obtained from $v(t)$ in (2.6) and it is exponentially vanishing.

Assumption 3.1. $\mathbf{A}(t)$ is almost everywhere time differentiable in any open interval $(t, t+$ $T$ ) except possibly at a finite set $c_{t}$ of isolated instants $t_{i} \in(t, t+T), i=1,2, \ldots, c_{t}$, where it is impulsive taking values $K_{i} \delta\left(t-t_{i}\right)$, so that $\left\|\sum_{i=1}^{c_{t}} k_{i}\right\| \leq \alpha$ (i.e., $\mathbf{A}\left(t_{i}\right)$ is discontinuous and then $\dot{\mathbf{A}}(t)$ is impulsive at $\left.t_{i} \in(t, t+T)\right)$. Furthermore, there exist real constants $\mu \in$ $\left[0, \mu^{*}\right), \alpha \geq 0$ for some $\mu^{*}>0$ such that for some real $T>0$ and all $t \geq 0$,

$$
\int_{t}^{t+T}\|\dot{\mathbf{A}}(t)(\tau)\|^{2} d \tau \leq \mu^{2} T+\alpha
$$

(or, alternatively, $\operatorname{Sup}_{t \geq 0}(\|\dot{\mathbf{A}}(t)\|) \leq \mu$, some $\mu \in\left[0, \mu^{*}\right)$ ). Thus, the system is globally exponentially stable (GES) if $\mu^{*}$ is sufficiently small.

Furthermore, note that $\mathbf{A}(t)$ is uniformly bounded and almost everywhere time differentiable in any open interval $(t, t+T)$ and it has bounded entries and eigenvalues in Res $\leq-\rho_{0}<0$ for all $t \geq 0$. This follows from Theorem 2.5 and the fact that the reference model is strictly stable with stability abscissa not exceeding $\left(-\rho_{0}\right)<0$ which accounts for possible stable plant cancellations included as poles of the reference model. The time instants where it is not differentiable are those where the estimation scheme switches between two estimators. There is a finite number of switching instants within any finite interval since each estimator parameterizes the adaptive controller subject to a minimum residence time. The intuitive implications of Assumption 3.1 are that the adaptation rate is sufficiently slow and that the residence time for each estimator to parameterize the controller, that is, the interval between any two consecutive estimator switches where $\dot{\mathbf{A}}(t)$ is impulsive, is sufficiently large. The following main stability result is proved in Appendix B by first obtaining appropriate global stability results related to the stability of the auxiliary system (3.8)-(3.10).

Theorem 3.2. The subsequent items hold.

(i) Assume that the plant (2.1) is uncertainty-free and perfectly known and that a strictly stable reference model of transfer function $1 / A_{m}\left(s, e^{-h s}\right)$ is set with $\partial A_{m k} \leq 2 n(k=$ $0,1, \ldots, m+1)$. Thus, if Assumptions 2.1(1)-(3) hold, then $y_{f}(t), u_{f}(t), y(t)$, and $u(t)$ are bounded for all time provided that $y^{*} \in L_{1}$. Furthermore, if $y^{*} \equiv 0$, then $y_{f}(t) \rightarrow 0$ as $t \rightarrow \infty, u_{f}(t) \rightarrow 0$ and $y(t) \rightarrow 0$ as $t \rightarrow \infty, u(t) \rightarrow 0$ exponentially as $t \rightarrow \infty$ or any bounded initial conditions. If the delay is unknown but the error between the true and measured delay is sufficiently small, then the closed-loop system is still globally exponentially stable (GES).

(ii) Assume that the plant is uncertainty-free with unknown parameters and subject to Assumptions 2.1(1)-(3), and 2.4. Assume also that the delay-free auxiliary system is GES. Thus, the adaptive controller based on the estimation algorithm (2.9)-(2.12) with a single estimation scheme (i.e., $p=1$ ) leads to a GES closed-loop system provided that the error between the true and measured delay in Assumption 2.1(4) and $\delta_{j}=\operatorname{Sup}_{t \geq 0}\left(\delta_{j}(t)\right)(j=1,2, \ldots, p)$ in Assumption 2.4 is both sufficiently small compared to the stability abscissa of the delay-free auxiliary system. The precise condition 
becomes alleviated if the above parallel multiestimation model (i.e., $p>1$ ) is used instead the single-estimation one. The results still hold if the auxiliary delay-free system is GES.

(iii) Assume that the plant is subject to uncertainties including unmodeled satisfying Assumption 2.2 and estimated from (2.13). Thus, the closed-loop system is globally stable (GS) under similar conditions as in (ii).

Remarks 3.3. (1) Note that $\widetilde{A}_{m}$ results from the fact that the set of solutions of $\left(R_{i k}, S_{i k}\right)$ of the diophantine equation causes, in general, the right-hand side in (3.6) distinct from $A_{m}$ since the solved unknowns $\left(R_{i k}, S_{i k}\right)$ for $k=0,1, \ldots, m$ and any $i$ th estimator involve powers $k=0,1, \ldots, 2 m$ of $e^{-h D}$ for $k=2 m+1$ to $2 q$.

(2) Note also that the closed-loop transfer function has (delay-free) spectrum under the reference model choice $A_{m}\left(s, e^{-h s}\right) \equiv A_{m 0}(s)$, where $A_{m 0}(s)$ is any strictly Hurwitzian polynomial.

(3) Finally, note in the case of known plants from Theorem 3.2(i) that if $1 /\left(A_{m}\left(s, e^{-h s}\right)\right.$ $\left.+\tilde{A}_{m}\left(s, e^{-h s}\right)\right)$ is stable, then the closed-loop system is stable even if the closed-loop spectrum is not prefixed to the zeros of $A_{m}\left(s, e^{-h s}\right)$. The precise "smallness" conditions referred to in Theorem 3.2(ii) and (iii) are explicitly established in a sufficiency context in the proofs in Appendix B for sufficiently large stability abscissa of the homogeneous system (3.8)-(3.10) provided that the adaptation rates of the estimates are sufficiently slow (Assumption 3.1).

\subsection{Summary and interpretation of the conditions that guarantee closed-loop expo- nential stability and stability and its monitoring through the adaptation process.}

3.4.1. $\mathbf{A}(t)$ (matrix of dynamics of the homogeneous auxiliary system). (a) It is a stability matrix with constant eigenvalues. This property is achieved since the designed reference model is stable and time-invariant and the pole-zero cancellations of the plant (if any) are stable, that is, the zeros of $\left(A_{m} E\right)$ lie strictly inside $\operatorname{Re} s \leq-\rho_{0}<0$. This is a necessary condition for the homogeneous auxiliary system to be exponentially stable with stability abscissa $(-\rho) \leq-\rho_{0}$, but since it is time-varying, it does not guarantee directly its exponential stability.

(b) Its entries are uniformly bounded from Theorem 2.5. They are also almost everywhere time differentiable since consecutive switching times between estimators of the parallel multiestimation scheme are subject to a minimum residence time. Since the homogeneous auxiliary system (3.8)-(3.10) is time-invariant, an extra condition to those given in (a) for guaranteeing exponential stability is that (3.11) holds with sufficiently small constant $\mu$. Such a constant is sufficiently small if the adaptation rate is sufficiently slow. This is monitored through the adaptation mechanism with sufficiently small constants $\alpha_{1}$ and $\alpha_{0}$ (see (2.12)-(2.13)).

3.4.2. Stability of the closed-loop system. It is guaranteed by that of the auxiliary inhomogeneous system as follows.

(a) The residence time for the parameterized adaptive controller at each estimate has to be sufficiently large compared to the $\alpha$ th constant (including the contribution of switches) 
in order that the inhomogeneous auxiliary system to be globally stable. See (B.33)-(B.34) in Appendix B.

(b) The stability abscissa of the homogeneous auxiliary system is sufficiently large compared to a threshold which depends on the number of delays, the maximum possible error in the measured base delay related to the true one, and the achievable upper bounds in the estimates of the delayed dynamics matrices $A_{k}$ (see Assumption 3.1) and the adjustable constant that estimates the contribution of the unmodeled dynamics (Assumption 2.2) with respect to the norm of the supreme of the auxiliary system state. The stability abscissa of the homogeneous auxiliary system may be increased through the choice of the reference model having sufficiently large stability degree when the plant has no cancellations. Otherwise, the stable cancellation establishes a maximum achievable value for the stability abscissa of the homogeneous auxiliary system. That constraint guarantees the stability of the inhomogeneous auxiliary system provided that the homogeneous one is exponentially stable so that the closed-loop stability of the proposed adaptive scheme is guaranteed. See, for instance, (B.15) and (B.17) in Appendix B. Note also from (B.33) and (B.34) that the residence time increases as the stability abscissa of the homogeneous system decreases and vice versa.

\section{Simulation example}

Consider the following nominal system $y(t)=\theta_{0}^{T} \varphi_{0}(t)+0.17 y(t-0.02)+\eta(t)$ with the undelayed part being defined by the parameter vector

$$
\theta=(1.68346,-1.02165,0.14512,1,0.245603,-0.278632,0.0464387)
$$

and the delay being $h^{*}=0.02$ second but unknown. The unmodeled dynamics is defined by the first-order unmodeled dynamics given by the input-output transfer function $0.1 /(s+20.8581)$. The suitable closed-loop polynomial is $A_{m}(s)=(s+6.02)\left[(s+7.4)^{2}+\right.$ $55.2]$. An adaptive regulator (i.e., $\left.y^{*}(t) \equiv 0\right)$ is designed with a biestimation scheme consisting of two estimation schemes (i.e., $r=2$ ). All the filters initialized to zero initial conditions and $F_{i}(D)=F(D)=D+19.48(i=1,2)$. The upper-bound of the unmodeled dynamics contribution is defined by constants $\varepsilon_{1 i}=1 ; \varepsilon_{2 i}=10^{-5}$, and $\sigma_{0 i}=0.1(i=1,2)$. The initial values of the estimates are

$$
\begin{aligned}
& \hat{\theta}_{1}^{T}(0)=(-0.005,-0.005,0,1,-0.008,-0.003), \\
& \hat{\theta}_{2}^{T}(0)=(-0.015,-0.015,0.5,10,-0.08,-0.03),
\end{aligned}
$$

and $P_{i}(0)=\operatorname{Diag}\left(10^{6}\right)$ for $i=1,2$. It is assumed that the base delay is unknown within the domain $[0,0.02]$. The first estimate assumes zero delay while the second one is based on using a delay $h^{\prime}=0.02$ second in its regressor. In this example, the various regressors are slightly modified with respect to the case of known delay so that different regressors, rather than a unique one, are used for each estimation algorithm in (2.12) according to 


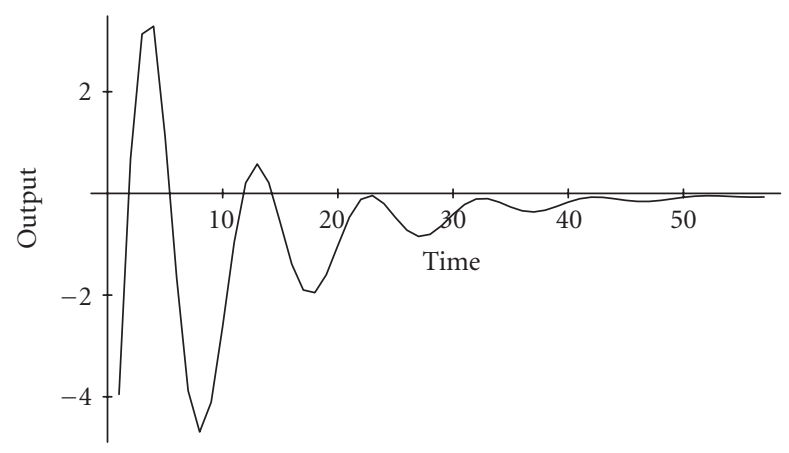

(a)

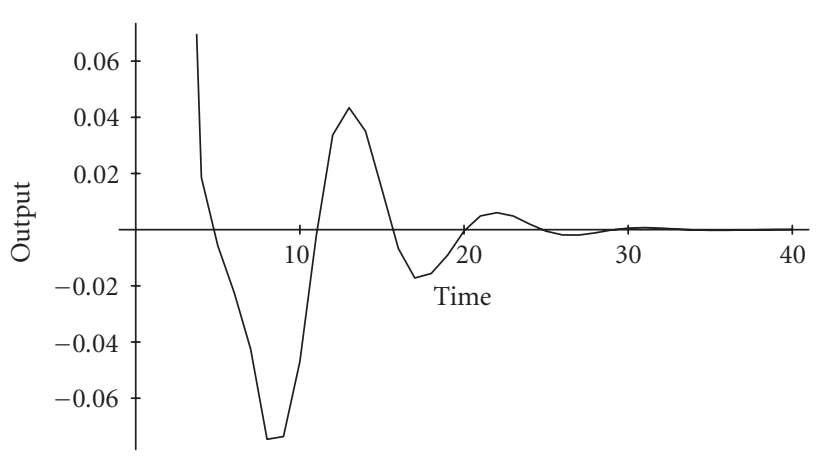

(b)

Figure 4.1. (a) Output for the first estimator only and (b) output for the combined estimator.

the assumed tentative delays. The residence time is $T_{r}=2, T_{0}=0.02$ second and the tuning error involves a delay being $h=T_{r}$. The output is displayed in Figure 4.1 for the use of the first estimator only or the combined one.

\section{Conclusions}

This paper has presented a robustly stable adaptive scheme for time-varying linear plants subject to a set of bounded point-delays. However, the delays are not required to be exactly known. Both the delay-free and delayed dynamics are possibly subject to timevarying disturbances, consisting of unmodeled dynamics and bounded noise, which grow at most linearly with the regressor norm of the parameter estimation scheme. However, the constants defining an overbounding function for the unmodeled dynamics contribution to the filtered output are not necessarily known precisely since they may be incorporated to the estimation scheme. Thus, the overbounding function required for adaptive stabilization purposes can be estimated as well. This paper has dealt with the robust adaptive control of a continuous linear and time-invariant plant possibly subject to bounded 
disturbances and a common class of unmodeled dynamics. The main novelty of the proposed scheme is that a multiestimation scheme with a parallel structure is involved. The control parameters are obtained for all time after switching from an estimation scheme to another one after accomplishing with a minimum residence time at the current estimator. Each estimator possesses its own input-related regressor vector. The switching mechanism consists of minimizing a loss function of the time integral of the square-tracking error over a receding finite-time-receding horizon. The main interest of such a synthesis philosophy relies on the case when there is no precise knowledge about a domain to which the true parameters belong but there is a set of possible domains available or on the case when there are changes in the plant operation point. Another interest which has been emphasized is that the multiestimation may be used to adjust the estimated delay closely to the true one with an judicious choice of the switching mechanism between estimation models. The robust closed-loop stabilization is achieved by using a relative adaptation dead zone which freezes the estimation in each estimation scheme for small tuning errors. In particular, the estimation process is frozen when the absolute tuning error is less than an appropriate available overbounding function of the contribution of the uncertainties to the filtered output. All the properties of the individual estimation schemes result to be identical to those being achievable from the use of a single estimator provided that a proper minimum residence time is used for the adaptive controller parameterization at each estimator. The closed-loop stability is investigated through that of an extended auxiliary system whose state is constructed by filtered plant input and output signals as well as their successive relevant time derivatives. The obtained main result is that robust adaptive closed-loop stability is achieved if the following features happen.

(1) The adaptation is performed at a sufficiently slow rate compared to the ratio of the maximum eigenvalue of the covariance gain matrix of the estimation scheme and the stability abscissa of the (closed-loop) delay-free disturbance-free estimation model. Furthermore, the switching actions between estimators to parameterize the adaptive controller are subject to a minimum residence time. This is the practical method which has been used to accomplish with Assumption 3.1. In particular, the eigenvalues of the auxiliary extended system (3.8)-(3.10) are constant and located in the open complex left-half plane.

(2) In the absence of identification error, the stability abscissa of the delay-free part of the estimation model is sufficiently large compared to the contributions of the delayed dynamics.

(3) The limit of the estimated constant slope of the above-mentioned overbounding function is sufficiently small compared to the stability abscissa of the extended delayed system for zero identification error. This point has been addressed in some intermediate results given in Appendix B to prove the closed-loop stability of the overall adaptive system. The above first intermediate property allows the maintenance of the closed-loop stability in the presence of delays and in the absence of identification error since the homogeneous auxiliary extended is proved to be globally exponentially stable if its delayfree part is exponentially stable. The second property is related to the tolerance to disturbances of the given scheme. It is found that such a tolerance is improved as the stability abscissa of the forced extended system becomes increased. 
(4) If a parallel multiestimation scheme is used, then there is a minimum residence time at each estimator (i.e., between consecutive estimation models), which depends on the parameterization and the stability abscissa of the delay-free system.

\section{Appendices}

\section{A. Properties of the estimation algorithms}

Proof of Theorem 2.5(i). Consider Lyapunov's-like candidate functions

$$
V_{i}(t)=\frac{1}{2}\left(\tilde{\theta}_{i}^{T}(t) P_{i}^{-1}(t) \tilde{\theta}_{i}^{T}(t)+\left(\widetilde{\varepsilon}_{i 1}^{2}(t)+\widetilde{\varepsilon}_{i 2}^{2}(t)\right)\right)
$$

for all $i=1,2, \ldots, p$. Taking time-derivatives using the estimation algorithm (2.9)-(2.13), $\dot{P}_{i}^{-1}(t)=-P_{i}^{-1}(t) \dot{P}_{i}(t) P_{i}^{-1}(t)$, and (2.8) by neglecting the asymptotically vanishing signal $v t$, one gets

$$
\begin{aligned}
& \dot{V}_{i}(t)=\tilde{\theta}_{i}^{T}(t) P_{i}^{-1}(t) \tilde{\theta}_{i}(t)-\frac{1}{2} \tilde{\theta}_{i}^{T}(t) P_{i}^{-1}(t) \dot{P}_{i}(t) P_{i}^{-1}(t) \tilde{\theta}_{i}(t)+\alpha_{1} \alpha_{0}^{-1}\left(\tilde{\varepsilon}_{i 1}(t) \dot{\widetilde{\varepsilon}}_{i 1}(t)+\tilde{\mathcal{\varepsilon}}_{i 2}(t) \dot{\widetilde{\varepsilon}}_{i 2}(t)\right) \\
& =b_{i}(t) \tilde{\theta}_{i}^{T}(t) \varphi(t) e_{i}(t)+\frac{1}{2} b_{i}(t)\left(\tilde{\theta}_{i}^{T}(t) \varphi(t)\right)^{2} \\
& +\frac{1}{2} b_{i}(t)\left(\tilde{\varepsilon}_{i 1}(t), \tilde{\varepsilon}_{i 2}(t)\right)\left(\operatorname{Sup}_{0 \leq \tau \leq t}\left(e^{-2 \rho_{0}(t-\tau)}\|\varphi(\tau)\|^{2}, 1\right)\right) T \leq-\frac{1}{2} b_{i}(t)\left(\gamma_{i l}(t)+\bar{\gamma}_{i}(t)\right)
\end{aligned}
$$

with

$$
\begin{gathered}
\gamma_{i l}^{T}(t)=\left(\tilde{\varepsilon}_{i 1}(t), \tilde{\varepsilon}_{i 2}(t)\right)\left(\operatorname{Sup}_{0 \leq \tau \leq t}\left(e^{-2 \rho_{0}(t-\tau)}\|\varphi(\tau)\|^{2}, 1\right)\right), \\
\bar{\gamma}_{i}(t)\left(\vartheta^{2}-1\right) \hat{\gamma}_{i}^{2}(t) \geq \frac{1}{2} b_{i}(t)\left(\frac{\vartheta^{2}-1}{\vartheta}\right) e_{i}^{2}(t) \geq 0 .
\end{gathered}
$$

Note that since the time-derivatives of the estimates of the $\varepsilon_{(\cdot)}$-constants are always nonnegative and their initial conditions are zero, $\gamma_{i l} \leq 0$ for all $t \geq t_{i 0}$ with $t_{i 0}=\operatorname{Max}\left(t_{i j} ; j=\right.$ $1,2)$ being such that $\widehat{\varepsilon}_{i j}(t) \geq \varepsilon_{j}$ for all $t \geq t_{i 0} ; i=1,2, \ldots, p$. It is obvious that such a finite time $t_{i 0}$ exists for all $i=1,2, \ldots, p$. Thus, $\dot{V}_{i}(t) \leq 0$ for all $t \geq t_{0 i}$ (some finite $t_{0 i}$ ) so that $V_{i} \in L_{\infty}$, if $\hat{\theta}_{i}(0)$ is bounded, it guarantees $\left\|\tilde{\theta}_{i}\right\| \in L_{\infty},\left\|\hat{\theta}_{i}\right\| \in L_{\infty}$ and $\left|\widetilde{\varepsilon}_{i j}\right| \in L_{\infty},\left|\hat{\varepsilon}_{i j}\right| \in$ $L_{\infty}(i=1,2, \ldots, p, j=1,2)$, and has nonnegative finite limits and $\left\|\tilde{\theta}_{i}\right\| \in L_{\infty}$. It also exists a finite $t_{0}=\operatorname{Max}\left(t_{i 0}, i=1,2, \ldots, p\right)$ such that all the $\dot{V}_{i}(t) \leq 0$ for all $t \geq t_{0}, i=1,2, \ldots, p$. Since $\left\|\theta_{i}\right\| \in L_{\infty}$ and $\gamma_{0} \in L_{\infty}$, thus, $b_{i}\left(\hat{\gamma}_{i}-e_{i}^{2}\right) \in L_{\infty}$ from (A.2), so that $\left(b_{i} \hat{\gamma}_{i}\right) \in L_{\infty}$ and $b_{i}^{1 / 2}\left|e_{i}\right| \in L_{\infty}$ since $\hat{\gamma}_{i}^{1 / 2}(t)>\left|e_{i}(t)\right|$ for $t \geq t_{0}$ (finite) if $b_{i}(t) \neq 0$ for all $i=1,2, \ldots, p$. Also, $b_{i}\left|\tilde{\theta}_{i}^{T} \varphi\right| \in L_{\infty}$, and, furthermore, $\dot{\hat{\theta}}_{i} \in L_{2} \cap L_{\infty}$ since $s_{i} \in L_{2} \cap L_{\infty}$ and $b_{i}\left\|P_{i} \varphi \varphi^{T}\right\| \in L_{\infty}$. Also, $b_{i}\left(\eta_{f}-e_{i}^{2}\right) \in L_{\infty}$ if $\vartheta>1(i=1,2, \ldots, p)$. Item (i) has been fully proved.

(ii) Since $\gamma_{1}(t) \geq 0$ and $\bar{\gamma}_{i}(t) \geq 0$ if $\hat{\varepsilon}_{i j \infty} \leq \varepsilon_{j}(i=1,2, \ldots, p, j=1,2)$ with possible switching-off the estimation of those constants, both terms that upper bound for all $t$ 
the time-derivatives of the Lyapunov functions in (A.2) are not positive. Thus,

$$
\begin{gathered}
b_{i}\left(\hat{\gamma}-e_{i}^{2}\right) \in L_{1} \cap L_{\infty}, \quad b_{i}^{1 / 2}\left(\hat{\gamma}_{i}^{1 / 2}\left|e_{i}\right|\right) \in L_{1} \cap L_{\infty}, \\
b_{i}^{1 / 2}\left(\hat{\gamma}_{i}^{1 / 2}\left|e_{i}\right|\right) \in L_{1} \cap L_{\infty}, \quad b_{i}^{1 / 2} \hat{\gamma}_{i}^{1 / 2} \in L_{1} \cap L_{\infty}, \quad b_{i}^{1 / 2}\left|e_{i}\right| \in L_{1} \cap L_{\infty} .
\end{gathered}
$$

Also, all the above signals converge asymptotically to zero as time tends to infinity. Also, $b_{i}\left(\eta_{f}-e_{i}\right)^{2} \in L_{1} \cap L_{\infty}$ and converges asymptotically to zero as time tends to infinity if $\vartheta>1$. The proof of (ii) is complete.

\section{B. Auxiliary stability results to prove Theorem $\mathbf{3 . 2}$}

Proof of Theorem 3.2. (i) From (3.5)-(3.6), the diophantine closed-loop equation for the time-invariant case of known parameters becomes

$$
A\left(D, e^{-h D}\right) R^{0}\left(D, e^{-h D}\right)+B\left(D, e^{-h D}\right) S\left(D, e^{-h D}\right)=A_{m}\left(D, e^{-h D}\right)+\widetilde{A}_{m}\left(D, e^{-h D}\right)
$$

or, equivalently,

$$
A\left(D, e^{-h D}\right) R\left(D, e^{-h D}\right)+B\left(D, e^{-h D}\right) S\left(D, e^{-h D}\right)=A_{m}\left(D, e^{-h D}\right),
$$

where $A_{m}\left(D, e^{-h D}\right)=\sum_{k=0}^{2 q} A_{m k}(D) e^{-k h D}$ with $\partial A_{m k} \leq 2 n(k=0,1, \ldots, m)$, since $R\left(D, e^{-h D}\right)=$ $-\tilde{A}_{m}\left(D, e^{-h D}\right) / A\left(D, e^{-h D}\right)$, and

$$
\begin{aligned}
A\left(D, e^{-h D}\right) R^{0}\left(D, e^{-h D}\right) & =\sum_{k=0}^{2 q} \sum_{\ell=\operatorname{Max}(0, k-n)}^{\operatorname{Min}(n, k)} A_{\ell}(D) R_{k-\ell}(D) e^{-k h D} \\
B\left(D, e^{-h D}\right) S\left(D, e^{-h D}\right) & =\sum_{k=0}^{2 q} \sum_{\ell=\operatorname{Max}(0, k-m)}^{\operatorname{Min}(m, k)} B_{\ell}(D) S_{k-\ell}(D) e^{-k h D} .
\end{aligned}
$$

Note that $S\left(s, e^{-h s}\right) / R\left(s, e^{-h s}\right)=S\left(s, e^{-h s}\right) A\left(s, e^{-h s}\right) /\left(A\left(s, e^{-h s}\right) R^{0}\left(s, e^{-h s}\right)-\widetilde{A}_{m}\left(s, e^{-h s}\right)\right)$ is a rational realizable (in general, transcendent) transfer function since $\operatorname{Max}\left[\partial_{s}(A R)\right] \geq$ $\operatorname{Max}\left[\partial_{s}(B S)\right]$ since $B / A$ and $S / R^{0}$ (and then $S / R$ ) are realizable. Now, combining the filtered output and control equations $y_{f}(t)=\left(B\left(D, e^{-h D}\right) / A\left(D, e^{-h D}\right)\right) u_{f}(t)$ and $u_{f}(t)=$ $\left(B\left(D, e^{-h D}\right) A\left(D, e^{-h D}\right)\right)\left(y_{f}^{*}(t)-y_{f}(t)\right)$ and using (B.1), one gets the closed-loop description

$$
\begin{aligned}
& A_{m}\left(D, e^{-h D}\right) y_{f}(t)=B\left(D, e^{-h D}\right) S\left(D, e^{-h D}\right) y_{f}^{*}(t), \\
& A_{m}\left(D, e^{-h D}\right) u_{f}(t)=A\left(D, e^{-h D}\right) S\left(D, e^{-h D}\right) y_{f}^{*}(t),
\end{aligned}
$$

which guarantees global internal closed-loop stability and yields directly the result.

Theorem 3.2(i) has been proved. Theorem 3.2((ii)-(iii)) are proved through the subsequent set of stability results. 
B.1. Auxiliary delay-free homogeneous system. The subsequent result guarantees that the auxiliary system (3.8)-(3.10) is GES under Assumptions 2.1-3.1.

Lemma B.1. Consider the nth time-varying homogeneous system $\dot{\mathbf{z}}(t)=\mathbf{A}(t) z(t)$, where

(1) $\mathbf{A}(t)$ has bounded entries and eigenvalues in $\operatorname{Re} s \leq-\rho_{0}$ for all $t \geq 0$;

(2) $\mathbf{A}(t)$ satisfies Assumption 3.1.

Proof. Since $\mathbf{A}(t)$ is a stability matrix for all $t \geq 0$, there is a unique symmetric positive definite matrix $Q(t)$ that satisfies Lyapunov's matrix equation:

$$
\mathbf{A}^{T}(t) Q(t)+Q(t) \mathbf{A}(t)=-I
$$

with $Q(t)=\int_{0}^{\infty} e^{A^{T}(t) \tau} e^{A(t) \tau} d \tau$ being everywhere time differentiable by construction and satisfying furthermore

$$
\mathbf{A}^{T}(t) \dot{Q}(t)+\dot{Q}(t) \mathbf{A}(t)=-\left(\dot{\mathbf{A}}^{T}(t) Q(t)+Q(t) \dot{\mathbf{A}}(t)\right)
$$

with $\dot{Q}(t)=\int_{0}^{\infty} e^{A^{T}(t) \tau}\left(\dot{\mathbf{A}}^{T}(t) Q(t)+Q(t) \mathbf{A}(t)\right) e^{\mathbf{A}(t) \tau} d \tau$ so that

$$
\lambda_{\max }(Q(t)):=\|Q(t)\|_{2} \leq \frac{k_{0}^{2}}{2 \rho_{0}}, \quad\|\dot{Q}(t)\|_{2} \leq \frac{K_{0}^{2}}{\rho_{0}} \lambda_{\max }(Q(t))\|\dot{A}(t)\|_{2} \leq \frac{K_{0}^{4}}{2 \rho_{0}^{2}}\|\dot{A}(t)\|_{2}
$$

since $Q(t)$ is symmetric positive definite and $\left\|e^{A(t) \tau}\right\|_{2} \leq \bar{K}_{0}(t) e^{-\bar{\rho}_{0} \tau} \leq K_{0} e^{-\rho_{0} \tau}$ for some $\bar{K}_{0}:[0, \infty] \rightarrow \mathbb{R}^{+}$and $\bar{\rho}_{0}:[0, \infty) \rightarrow \mathbb{R}^{+}$upper and lower bounded by $K_{0}$ and $\rho_{0}$, respectively, since $\mathbf{A}(t)$ is bounded with negative stability abscissa and $Q(t)$ is symmetric positive definite for all $t \geq 0$ so that

$$
\beta \leq \lambda_{\min }(Q(t)) \leq \lambda_{\max }(Q(t)) \leq \beta_{2}
$$

Now, consider Lyapunov's function candidate $V(t)=z^{T}(t) Q(t) z(t)$ of time derivative:

$$
\begin{aligned}
\dot{V}(t) & =z^{T}(t)\left[\dot{Q}(t)+\mathbf{A}^{T}(t) Q(t)+Q(t) \mathbf{A}(t)\right] z(t) \leq\|z(t)\|_{2}^{2}+\left|z^{T}(t) \dot{Q}(t) z(t)\right| \\
& \leq-\left(1-c\|\dot{\mathbf{A}}(t)\|_{2}\right)\|z(t)\|_{2}^{2}
\end{aligned}
$$

where $c=K_{0}^{4} / 2 \rho_{0}^{2}$. On the other hand,

$$
\beta_{1}^{-1} V(t) \geq \lambda_{\min }^{-1}(Q(t)) V(t) \geq\|z(t)\|_{2}^{2} \geq \lambda_{\max }^{-1}(Q(t)) V(t) \geq \beta_{2}^{-1} V(t)
$$

Thus, one gets from (B.8) and (B.9),

$$
\begin{gathered}
\dot{V}(t) \leq-\left(1-c\|\dot{\mathbf{A}}(t)\|_{2}\right) \beta_{2}^{-1} V(t) \quad \text { if } 1>c \operatorname{Sup}_{t \geq 0}\left(\|\dot{\mathbf{A}}(t)\|_{2}\right), \\
\dot{V}(t) \leq-\left(\beta_{2}^{-1}-c\|\dot{\mathbf{A}}(t)\|_{2} \beta_{1}^{-1}\right) V(t)
\end{gathered}
$$


otherwise. Thus, from (B.10), one gets

$$
\begin{aligned}
\|z(t)\|_{2}^{2} & \leq \beta_{1}^{-1} V(t) \leq \beta_{1}^{-1} \exp \left\{-\int_{0}^{t}\left(\beta_{2}^{-1}-c \beta_{1}^{-1}\right)\|\dot{\mathbf{A}}(\tau)\|_{2} d \tau\right\} V(0) \\
& \leq \beta_{1}^{-1} \beta_{2} \exp \left(-\beta_{2}^{-1} t\right) \exp \left\{c \beta_{1}^{-1}\left[\mu^{2} t^{2}+\alpha t\right]^{1 / 2}\right\}\|z(0)\|_{2}^{2}
\end{aligned}
$$

since

$$
\int_{t}^{t+T}\|\dot{\mathbf{A}}(\tau)\|_{2} d \tau \leq\left(\int_{t}^{t+T}\|\dot{\mathbf{A}}(\tau)\|_{2}^{2} d \tau\right)^{1 / 2} \sqrt{T} \leq\left(\mu^{2} T+\alpha\right)^{1 / 2} \sqrt{T} \leq \mu \sqrt{T}+\sqrt{\alpha}
$$

Now, assume that $\mu^{*}<(1 / 2)\left(\beta_{1} / c \beta_{2}\right)=\left(\beta_{1} / \beta_{2}\right)\left(\rho_{0} / K_{0}\right)^{2}$, then $\|z(t)\|_{2}^{2} \leq(1 / 2)\left(\alpha c^{2} \beta_{2}^{2} /\right.$ $\left.\beta_{1}^{3}\right) \exp (-\rho t)\|z(0)\|_{2}^{2} \rightarrow 0$ exponentially as $t \rightarrow \infty$ with $\rho=(1 / 2) \beta_{2}^{-2}-c \mu \beta_{1}^{-1}$ provided that $\mu \in\left[0, \mu^{*}\right)$. Similarly, if $\|\dot{\mathbf{A}}(t)\|_{2}$ is bounded with $1>c \operatorname{Sup}_{t \geq 0}\left(\|\dot{\mathbf{A}}(t)\|_{2}\right)=c \mu=\left(K_{0}^{4} / 2 \rho_{0}^{2} \mu\right)$.

Thus, $\dot{V}(t) \leq-\delta_{a} V(t)$ with $\delta_{a}=\beta_{2}^{-1} \mathcal{E}^{\prime}=\beta_{2}^{-1}\left(1-c \operatorname{Sup}_{t \geq 0}\left(\|\dot{\mathbf{A}}(t)\|_{2}\right)\right)$ so that $V(t) \leq$ $-e^{\delta_{a} t} V(0)$ and $\|z(t)\|_{2}^{2} \leq \beta_{1}^{-1} \beta_{2} \exp \left(\beta_{2}^{-} 1 \varepsilon t\right)\|z(0)\|_{2}^{2} \rightarrow 0$ exponentially as $t \rightarrow \infty$ with $\varepsilon=$ $1-\left(K_{0}^{4} / 2 \rho_{0}^{2}\right) \mu$ and the result has been fully proved.

B.2. Auxiliary time-delay inhomogeneous system. The subsequent result is related to the stability of the inhomogeneous auxiliary system (3.8)-(3.10) for the closed-loop description provided that the homogeneous delay-free system is subject to Lemma B.1 and Assumptions 2.1-2.2 hold. In other words, if the error between the true and estimated delays is sufficiently small, the true and parameter estimates lie within a region where the matrices associated with the delayed dynamics have sufficiently small norms. It is assumed, in addition, that the forcing signal grows not faster than linearly with

$$
o\left[\operatorname{Sup}_{t-q(h+\Delta h) \leq \tau \leq t}(\|x(\tau)\|)\right]
$$

with sufficiently small slope.

Lemma B.2. The following two items hold.

(i) Consider the time-varying inhomogeneous system

$$
\dot{x}(t)=\sum_{k=0}^{q} A_{k}(t) x(t-k h)+f(t)
$$

with initial conditions defined by the absolutely continuous vector function $\varphi:[-q h, 0] \rightarrow \mathbb{R}^{\mathbf{n}}$ except possibly at set of zero measure of bounded discontinuities. Assume the following.

(1) $\mathbf{A}(t)=\sum_{k=0}^{q} A_{k}(t)$ satisfies all the assumptions of Lemma B.1, that is, Assumption 3.1, and it is strictly stable of constant eigenvalues for all time, and $A_{k}(t)(k=1,2, \ldots$, q) has bounded entries on $[0, \infty)$.

(2) The base true delay $h$ is unknown but a maximum error $\pm \Delta h$ related to a nominal value is known with a maximum error of $(\Delta h \bullet 0)$. 
22 Adaptive control of systems with small delays

(3) $|f(t)|=o\left[\operatorname{Sup}_{t-q(h+\Delta h) \leq \tau \leq t}(\|x(\tau)\|)\right]$. Thus, the system (B.14) is GES if

$$
\rho>K e^{q \bar{h}}\left[2 a(1+q \Delta h b)+M_{0}\right]
$$

where $M_{0} \geq 0$ is such that $|f(t)| \leq M_{0} \operatorname{Sup}_{t-q \bar{h} \leq \tau \leq t}(\|x(\tau)\|)$ with $\bar{h}=h+\Delta h$, and

$$
a=\operatorname{Sup}_{t \geq 0}\left(\sum_{k=1}^{q}\left\|A_{k}(t)\right\|\right), \quad b=\operatorname{Sup}_{t \geq 0}\left(\sum_{k=0}^{q}\left\|A_{k}(t)\right\|\right) \leq a+\operatorname{Sup}_{t \geq 0}\left(\left\|A_{0}(t)\right\|\right) .
$$

Also, the system (B.14) is GES if

$$
\rho^{\prime}>K^{\prime} e^{q \bar{h}}\left[a(1+2 q b \Delta h)+M_{0}\right]
$$

provided that $\dot{z}_{0}(t)=A_{0}(t) z_{0}(t)$ is GES with its fundamental matrix satisfying $\left\|\Psi^{\prime}(t, \tau)\right\| \leq$ $K^{\prime} e^{-\rho^{\prime}(t-\tau)}$ for some real constants $K^{\prime} \geq 1$ and $\rho^{\prime}>0$.

(ii) Let now $f(t)$ be $f(t)=0\left[\operatorname{Sup}_{0 \leq \tau \leq t}(\|x(\tau)\|)\right]$. Thus, the system (B.14) is GS if $\rho>$ $K\left\lfloor 2 a(1+q b \Delta h)+M_{0}\right\rfloor$ provided that the homogeneous $\dot{z}(t)=\mathbf{A}(t) z(t)$ is GES. Also, (B.14) is GS if the homogeneous system $\dot{z}_{0}(t)=A_{0}(t) z_{0}(t)$ is GES and $\rho^{\prime}>K^{\prime}\left[a(2+q b \Delta h)+M_{0}\right]$.

Proof. (i) Equation (B.14) may be rewritten equivalently as

$$
\dot{x}(t)=\mathbf{A}(t) x(t)+\sum_{k=1}^{q} A_{k}(t)\left(x\left(t-k h^{\prime}\right)-x(t)\right)+\sum_{k=1}^{q} A_{k}(t)\left(x(t-k h)-x\left(t-k h^{\prime}\right)\right)+f(t)
$$

and since $\|\Psi(t, \tau)\| \leq K e^{-\rho(t-\tau)}$ (Lemma B.1) one gets by taking $\ell_{2}$-matrix and vector norms

$$
\begin{aligned}
\|x(t)\| \leq K e^{-\rho t} & {\left[\|x(0)\|+\left\|\int_{0}^{q \bar{h}} e^{\rho \tau}\left[\sum_{k=1}^{q} A_{k}(\tau)(x(\tau-k h)-x(\tau))+M_{0}\right] d \tau\right\|\right.} \\
& \left.+\int_{q \bar{h}}^{t} e^{\rho \tau}\left[\left(2 a(1+q b \Delta h)+M_{0}\right) \operatorname{Sup}_{\substack{\tau-q \bar{h} \leq \tau^{\prime} \leq \tau\\
}}\left(\left\|x\left(\tau^{\prime}\right)\right\|\right)\right]\right] d \tau^{\prime}
\end{aligned}
$$

since

$$
\begin{aligned}
\left\|x(t-k h)-x\left(t-k h^{\prime}\right)\right\| & \leq \int_{t-k h}^{t-k(h-\Delta h)}\left\|\dot{x}\left(\zeta_{k}(t-k h+\tau)\right) d \tau\right\| \\
& \leq \int_{0}^{q \Delta h} \operatorname{Sup}_{t-q \bar{h} \leq \tau \leq t-h+\Delta h}(\|\dot{x}(\tau)\|) d \tau \leq q \Delta h, \\
\left\|x(t-k h)-x\left(t-k h^{\prime}\right)\right\| & \leq\left\|\int_{t-k(h-\Delta h)}^{t-k(h+\Delta h)} \dot{x}\left(\zeta_{k}(\tau) d \tau\right)\right\| \leq \int_{t-q(h-\Delta h)}^{t-q(h+\Delta h)} \operatorname{Sup}_{t-q \bar{h} \leq \tau \leq t}(\|x(\tau)\|) d \tau \\
& \leq 2 q b \Delta h \operatorname{Sup}_{t-q \bar{h} \leq \tau \leq t}(\|x(\tau)\|),
\end{aligned}
$$


some $\zeta_{k}(\tau) \in(t-k \bar{h}, t-k(h-\Delta h))$, where La Rolle mean value theorem for integrals has been applied in the above first inequality. Thus, (B.19) leads to

$$
\|x(t)\| \leq K e^{-\rho t}\left(m+M \int_{q \bar{h}}^{t} e^{\rho \tau} \operatorname{Sup}_{t-q \bar{h} \leq \tau^{\prime} \leq \tau}\left(\left\|x\left(\tau^{\prime}\right)\right\|\right) d \tau^{\prime}\right)
$$

for $t \geq q \bar{h}$, where

$$
m=\|x(0)\|+\left\|\int_{0}^{q \bar{h}} e^{\rho \tau} \sum_{k=1}^{q} A_{k}(\tau)\left[(x(\tau-k h)-x(\tau))+M_{0}\right] d \tau\right\|, \quad M=2 a(1+q b \Delta h)+M_{0} .
$$

Define $\sigma(t)=e^{\rho t} \operatorname{Sup}_{t-q \bar{h} \leq \tau \leq t}(\|x(\tau)\|)$ for all $t \geq 0$ with $x(t) \equiv \varphi(t)$ for $t \in[-q \bar{h}, 0]$. Then, define $t^{\prime}(t)=\operatorname{Max}\left[t-q \bar{h} \leq \tau \leq t:\left\|x\left(t^{\prime}\right) \equiv \operatorname{Sup}_{t-q \bar{h} \leq \tau \leq t}(\|x(\tau)\|)\right\|\right]$ so that one gets from (B.21)

$$
\sigma(t) \leq K e^{\rho\left(t-t^{\prime}\right)}\left(m+M \int_{0}^{t} \sigma(\tau) d \tau\right)
$$

and Gronwall's Lemma yields directly since $t-t^{\prime} \leq q \bar{h}$,

$$
\sigma(t) \leq K e^{q \bar{h}} m \exp \left(K e^{q \bar{h}} M_{0} t\right)
$$

so that

$$
\|x(t)\| \leq \operatorname{Sup}_{t-q \bar{h} \leq \tau \leq t}(\|x(\tau)\|) \leq K e^{q \bar{h}} m \exp \left[-\left(\rho-K e^{q \bar{h}} M\right) t\right\rfloor
$$

which converges to zero exponentially as $t \rightarrow \infty$ if $\rho>K e^{q \bar{h}} M$ from (B.24) and thus the system is GES if (B.19) holds. If $\dot{z}_{0}(t)=A_{0} z_{0}(t)$ is GES with its fundamental matrix satisfying $\left\|\Psi^{\prime}(t, \tau)\right\| \leq K^{\prime} e^{-\rho^{\prime}(t-\tau)}$ for some real constants, $K^{\prime} \geq 1$ and $\rho^{\prime}>0$. Thus, (B.14) is identical to

$$
\dot{x}(t)=A_{0}(t) x(t)+\sum_{k=1}^{q} A_{k}(t) x\left(t-k h^{\prime}\right)+\sum_{k=1}^{q} A_{k}(t)\left(x\left(t-k h^{\prime}\right)-x(t-k h)\right)+f(t)
$$

to yield in the same way as above

$$
\|x(t)\| \leq K^{\prime} e^{-\rho^{\prime} t}\left[m+\int_{0}^{q \bar{h}} e^{\rho \tau}\left[\sum_{k=1}^{q}\left\|A_{k}(\tau)\right\|+2 q b \Delta h+M_{0}\right]\right] \operatorname{Sup}_{\tau-q \bar{h} \leq \tau^{\prime} \leq \tau}\left(\left\|x\left(\tau^{\prime}\right)\right\|\right) d \tau^{\prime}
$$

which has the same structure as (B.15) by replacing $K \rightarrow K^{\prime}, \rho \rightarrow \rho^{\prime}, M \rightarrow M^{\prime}=a(1+$ $2 q b \Delta h)+M_{0}$ so that the system is GES if (B.17) holds. 
(ii) Note from (B.14) that there exist real constants $0 \leq M_{0}<\infty$ and $0 \leq M_{0}^{\prime}<\infty$ such that

$$
\begin{gathered}
f(t)=O\left[\operatorname{Sup}_{t-t_{\alpha} \leq \tau \leq t}(\|x(\tau)\|)\right]=M_{0} \operatorname{Sup}_{0 \leq \tau \leq t}(\|x(\tau)\|)+M_{0}^{\prime}, \\
\|x(t)\| \leq K e^{-\rho t}\left(m+\left[2 a(1+q b \Delta h)+M_{0}\right]\right)\left(\int_{q \bar{h}}^{t} e^{\rho \tau} d \tau\right) \operatorname{Sup}_{0 \leq \tau \leq t}(\|x(\tau)\|)+\frac{K M_{0}^{\prime}}{\rho} \\
\leq \mathrm{Ke}^{-\rho t} m+K\left(\frac{2 a(1+q b \Delta h)+M_{0}}{\rho}\right) \operatorname{Sup}_{0 \leq \tau \leq t}(\|x(\tau)\|)+\frac{K M_{0}^{\prime}}{\rho} .
\end{gathered}
$$

Now, note that for each $t$, there exists $t^{\prime}(t)=\operatorname{Max}\left(\tau \leq t:\left\|x\left(t^{\prime}\right)\right\|=\operatorname{Sup}_{0 \leq \tau \leq t}(\|x(\tau)\|)\right)$ so that from (B.29), one gets

$$
\operatorname{Sup}_{0 \leq \tau \leq t}(\|x(\tau)\|) \leq K\left(\varepsilon_{0}^{-1} m e^{-\rho t^{\prime}}+\frac{M_{0}^{\prime}}{\rho}\right) \leq K\left(\frac{m}{\varepsilon_{0}}+\frac{M_{0}^{\prime}}{\rho}\right)<\infty
$$

with $\varepsilon_{0}=1-\left(2 a K(1+q b \Delta h)+M_{0}\right) / \rho>0$ and (B.14) is GS if $1>K\left\lfloor 2 a(1+q b \Delta h)+M_{0}\right\rfloor / \rho$. Alternatively, if the equivalent system description (B.26) is used, then a similar reasoning establishes that the system (B.14) is GS if $1>K^{\prime}\left[a(2+q b \Delta h)+M_{0}\right] / \rho^{\prime}$.

B.3. Particular results obtained from Lemma B.2. (1) If $h=h^{\prime}$ (i.e., $\Delta h=0$ ), then (B.14) is GES if $h<(1 / q) \ln \left(\rho / K\left(2 a+M_{0}\right)\right)$ provided that $\rho>K\left(2 a+M_{0}\right)$.

(2) If $h$ satisfies the above inequality, then $\Delta h<\Delta \bar{h}$ if $\Delta h<\Delta \bar{h}>0$ and $\Delta h=\Delta \bar{h}=0$, otherwise, where

$$
\Delta \bar{h}=\operatorname{Inf}_{\alpha \in[0,1]}\left(\frac{1}{q}\left[|\ln \alpha|, \frac{(\rho / K) \alpha e^{-q h}-M_{0}-2 a}{2 a b}\right]\right)
$$

provided that $\rho>K\left(2 a+M_{0}\right) e^{q h} / \alpha$. This holds since $(\rho / K) e^{-q h} e^{-q \Delta h}>M=M_{0}+2 a(1+$ $q b \Delta h)$. Very close results apply to the case when $\dot{z}_{0}(t)=A_{0}(t) z_{0}(t)$ is GES.

\section{B.4. Stability under multiestimation models.}

Lemma B.3. Assume that a multiestimation model is used and that all assumptions of Lemmas B.1-B.2 hold. Thus, Lemmas B.1-B.2 still hold if there is a minimum residence time for each estimator (i.e., if both the homogeneous and inhomogeneous systems are GES).

Proof. First note from Lemma B.1 that $\|\Psi(t, 0)\|_{2}=\operatorname{Max}_{\|z(0)\|_{2}}\left(\|\Psi(t, 0)\|_{2}\right) \leq(1 / 2) c\left(\beta_{2} /\right.$ $\left.\beta_{1}^{3 / 2} e^{-\rho t}\right)$ so that $K \sqrt{1 / 2} c \alpha\left(\beta_{2} / \beta_{1}^{3 / 2}\right)$ for the $\ell_{2}$-matrix norm. Let $t_{i+1}$ and $t_{i}$ be two consecutive switches between estimation models with $t_{i+1}-t_{i}=T_{i}$. Any switching of the estimator at time $t=t_{i}$ causes the modification $\int_{t}^{t+T_{i}}\|\dot{\mathbf{A}}(\tau)\|^{2} d \tau \leq \mu^{2} T_{i}+\alpha+\alpha_{i}$ to be considered in the developments of Lemma B.1, provided that no switches take place in $\left(t_{i}^{+}, t+T_{i}\right)$ while only the referred one occurs in $\left(t_{i}, t+T_{i}\right)$ with $\mathbf{A}(t)=\sum_{k=0}^{q} A_{k}(t)$, and

$$
\alpha=\operatorname{Max}_{i \geq 0}\left(\alpha_{i}\right) ; \quad \alpha_{i}=\left\|\sum_{k=0}^{q} A_{k}\left(t_{i}^{+}\right)-A_{k}\left(t_{i}\right)\right\| \leq 2 q a .
$$


Thus, the fundamental matrix $\Psi(t, \tau)$ of $A(t)$ satisfies $\|\Psi(t, \tau)\| \leq K_{i} e^{-\rho(t-\tau)}$ for $\tau \in\left[0, T_{i}\right]$ with $K_{i}^{2}=(1 / 2) c^{2}\left(\beta_{2}^{2} / \beta_{1}^{3}\right)\left(\alpha+\alpha_{i}\right)$. Thus, $\left\|z\left(t_{i+1}\right)\right\|_{2} \leq \lambda\left\|z\left(t_{i}\right)\right\|_{2}$ for any given real constant $\lambda \in(0,1)$ and all consecutive switching times $t_{i}$ between estimation models belonging to the sequence $S$ satisfy $t_{i+1}=t_{i}+T_{i}$ if $\|\Psi(t, \tau)\| \leq K_{i} e^{-\rho(t-\tau)} \leq \lambda$ that holds if the interval between consecutive switches satisfies

$$
T_{i} \geq \frac{1}{\rho}\left[\frac{1}{2} \ln \left(\alpha+\alpha_{i}\right)+\ln \left(\frac{c \beta_{2}}{\sqrt{2} \beta_{1}^{3 / 2}}\right)+\ln |\lambda|\right]
$$

that is guaranteed if $T_{i} \geq T$ (minimum residence time) that satisfies

$$
T \geq \frac{1}{\rho}\left[\frac{1}{2} \ln (\alpha+2 q a)+\ln c+\ln \beta_{2}-\frac{3}{2} \ln \beta_{1}-\frac{1}{2} \ln 2+|\ln \lambda|\right] .
$$

Thus, the inhomogeneous system (B.26) with switches between estimation models is GES if, in addition to the assumptions of Lemma B.1, there is a minimum residence time between any two consecutive switches subject to (B.33)-(B.34). Now, let $\lambda_{i}(i=$ $1,2, \ldots, p)$ and $\rho^{\prime}$ satisfy $K_{i} e^{-\rho T_{i}} \leq \lambda_{i}=e^{-\rho^{\prime} T_{i}}<1(i=1,2, \ldots, p)$ so that $\rho^{\prime}=\ln \left|\lambda_{i}\right| / T_{i}=$ $\ln |\lambda| / T_{i} \leq \rho$. Thus, the inhomogeneous system (B.26) is GES if Lemma B.2 holds under similar conditions by replacing $\rho \rightarrow \rho^{\prime}, K^{2} \rightarrow K_{i}^{2}=(1 / 2)\left(\alpha+\alpha_{i}\right)\left(\beta_{2}^{2} / \beta_{1}^{3}\right)\left(\right.$ or $K^{2} \rightarrow$ $(1 / 2)(\alpha+2 q a) c^{2}\left(\beta_{2}^{2} / \beta_{1}^{3}\right)$ for $i=1,2, \ldots, p$.

Proof of Theorem 3.2(ii)-(iii). Note that $\mathbf{A}(t)$ is bounded from Theorem 2.5 and it is almost everywhere time differentiable except possibly at isolated switching times and with constant eigenvalues of negative real parts for all time. Thus, Assumption 3.1 and all the assumptions of Lemma B.1 hold so that the delay-free auxiliary system (3.8)-(3.10) is GES. Then, Theorem 3.2(ii) follows directly from Lemma B.2, related to the single estimation scheme and Lemma B.3 related to the multiestimation scheme. Theorem 3.2(iii) follows from Lemma B.2(ii) for single estimation and Appendix B.4 for multiple estimation.

\section{Acknowledgments}

The authors are very grateful to MCYT for its partial support of this work via Project DPI 2003-0164 and to UPV for its support through Grant 1/UPV- EHU/I06.I006-EB $8235 / 2000$.

\section{References}

[1] A. Bilbao-Guillerna, M. De la Sen, A. Ibeas, and S. Alonso-Quesada, Robustly stable multiestimation scheme for adaptive control and identification with model reduction issues, Discrete Dynamics in Nature and Society 2005 (2005), no. 1, 31-67.

[2] T. A. Burton, Stability and Periodic Solutions of Ordinary Differential and Functional Equations, Academic Press, New York, 1987.

[3] Y.-Y. Cao and Y.-X. Sun, Robust stabilization of uncertain systems with time-varying multistate delay, IEEE Transactions on Automatic Control 43 (1998), no. 10, 1484-1488.

[4] H.-F. Chen and X.-R. Cao, Controllability is not necessary for adaptive pole placement control, IEEE Transactions on Automatic Control 42 (1997), no. 9, 1222-1229. 
[5] M. De la Sen, On some structures of stabilizing control laws for linear and time-invariant systems with bounded point delays and unmeasurable states, International Journal of Control 59 (1994), no. 2, 529-541.

[6] _ Adaptive controller for continuous systems with single internal and external delays, Dynamics and Control 6 (1996), no. 4, 387-403.

[7] _ On the robust adaptive stabilization of a class of nominally first-order hybrid systems, IEEE Transactions on Automatic Control 44 (1999), no. 3, 597-602.

[8] _ Allocation of poles of delayed systems related to those associated with their undelayed counterparts, Electronics Letters 36 (2000), no. 4, 373-374.

[9] M. De la Sen and A. Bilbao-Guillerna, Fractional hold circuits versus positive realness of discrete transfer functions, Discrete Dynamics in Nature and Society 2005 (2005), no. 3, 373-378.

[10] M. De la Sen and J. Jugo, Robust direct adaptive control for a class of systems with delays, IMA Journal of Mathematical Control and Information 15 (1998), no. 1, 25-52.

[11] M. De la Sen and A. Peña, Synthesis of controllers for arbitrary pole-placement in discrete plants including unstable zeros with extensions to adaptive control, Journal of the Franklin Institute $\mathbf{3 3 5}$ B (1998), no. 3, 471-502.

[12] V. Etxebarria and M. De la Sen, Adaptive control based on special compensation methods for timevarying systems subject to bounded disturbances, International Journal of Control 61 (1995), no. 3, 667-694.

[13] A. Feliachi and A. Thowsen, Memoryless stabilization of linear delay-differential systems, IEEE Transactions on Automatic Control 26 (1981), no. 2, 586-587.

[14] G. Feng, Analysis of a new algorithm for continuous-time robust adaptive control, IEEE Transactions on Automatic Control 44 (1999), no. 9, 1764-1768.

[15] P. A. Ioannou and J. Sun, Robust Adaptive Control, Prentice-Hall, New Jersey, 1996.

[16] E. W. Kamen, P. P. Khargonekar, and A. Tannenbaum, Stabilization of time-delay systems using finite-dimensional compensators, IEEE Transactions on Automatic Control 30 (1985), no. 1, 7578.

[17] J. H. Lee, S. W. Kim, and W. H. Kwon, Memoryless $H^{\infty}$ controllers for state delayed systems, IEEE Transactions on Automatic Control 39 (1994), no. 1, 159-162.

[18] J. Lu and Y. Yahai, New design methods for modified reference adaptive control for nonminimum phase systems with disturbances using approximate inverse systems, IEE Proceedings Control Theory and Applications 120 (1998), 117-124.

[19] T. Mori, E. Noldus, and M. Kuwahara, A way to stabilize linear systems with delayed state, Automatica 19 (1983), no. 5, 571-573.

[20] K. S. Narendra and J. Balakrishnan, Improving transient response of adaptive control systems using multiple models and switching, IEEE Transactions on Automatic Control 39 (1994), no. 9, 18611866.

[21] _ Adaptive control using multiple models, IEEE Transactions on Automatic Control 42 (1997), no. 2, 171-187.

[22] S.-I. Niculescu, Delay Effects on Stability. A Robust Control Approach, Lecture Notes in Control and Information Sciences, vol. 269, Springer, London, 2001.

[23] S.-I. Niculescu, J.-M. Dion, and L. Dugard, Robust stabilization for uncertain time-delay systems containing saturating actuators, IEEE Transactions on Automatic Control 41 (1996), no. 5, 742 747.

[24] C. Sampson and J. J. Fuchs, Discrete adaptive regulation of nonnecessarily minimum phase systems, Proceedings of IEE 128 (1991), no. 3, part D, 102-108.

[25] S. Sastry and M. Bodson, Adaptive Control: Stability, Convergence and Robustness, Prentice-Hall, New Jersey, 1989. 
[26] J.-C. Shen, B.-S. Chen, and F. C. K'ung, Memoryless stabilization of uncertain dynamic delay systems: Riccati equation approach, IEEE Transactions on Automatic Control 36 (1991), no. 5, 638-640.

[27] P. V. Zhivoglyadov, R. H. Middleton, and M. Fu, Localization based switching adaptive control for time-varying discrete-time systems, Proceedings of the 36th IEEE Conference on Decision and Control, vol. 5, California, December 1997, pp. 4151-4156.

M. De la Sen: Instituto de Investigación y Desarrollo de Procesos, Facultad de Ciencias, Universidad del País Vasco, Aparatado 644 de Bilbao, 48080 Leioa(Bizkaia), Spain E-mail address: wepdepam@lg.ehu.es

S. Alonso: Instituto de Investigación y Desarrollo de Procesos, Facultad de Ciencias, Universidad del País Vasco, Aparatado 644 de Bilbao, 48080 Leioa(Bizkaia), Spain E-mail address: santi@we.lc.ehu.es 


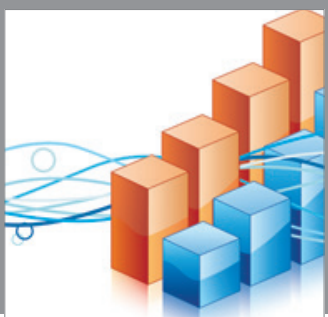

Advances in

Operations Research

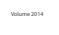

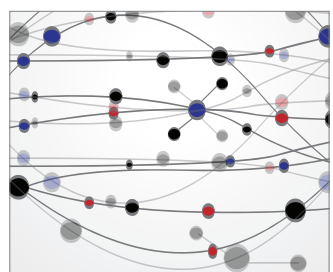

\section{The Scientific} World Journal
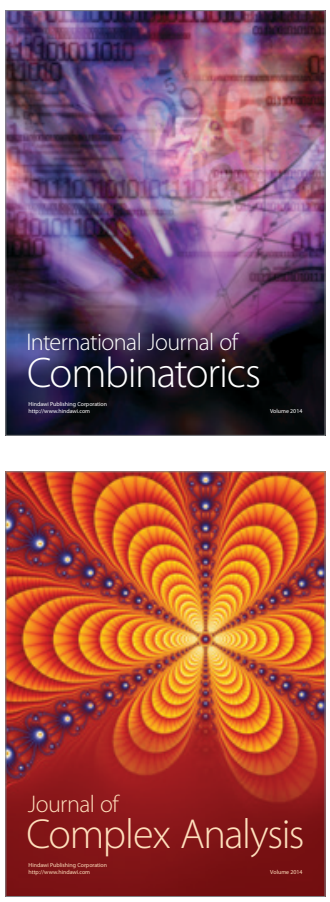

International Journal of

Mathematics and

Mathematical

Sciences
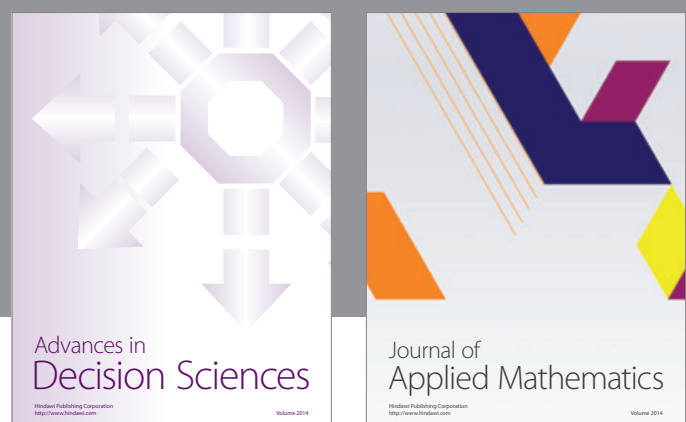

Journal of

Applied Mathematics
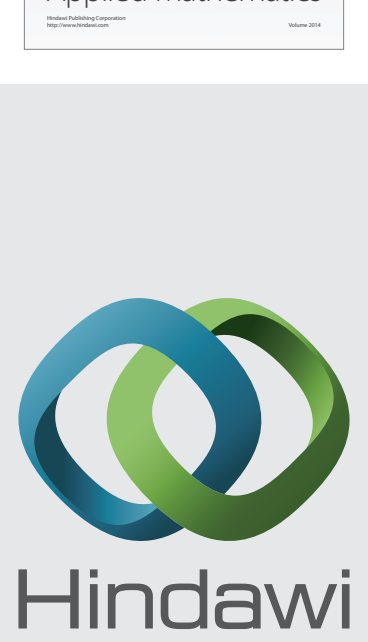

Submit your manuscripts at http://www.hindawi.com
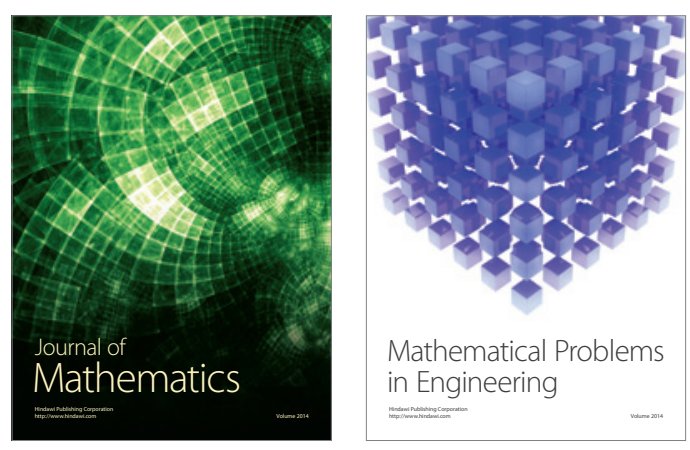

Mathematical Problems in Engineering
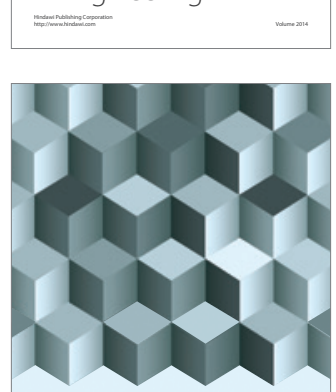

Journal of

Function Spaces
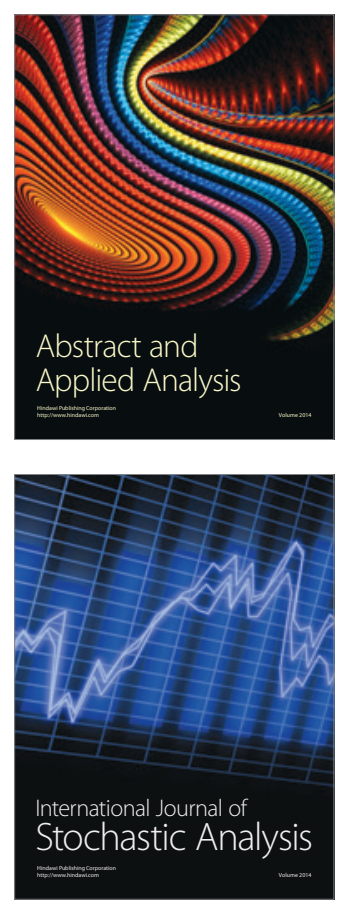

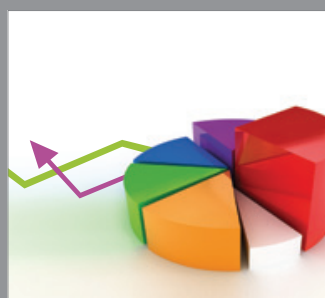

ournal of

Probability and Statistics

Promensencen
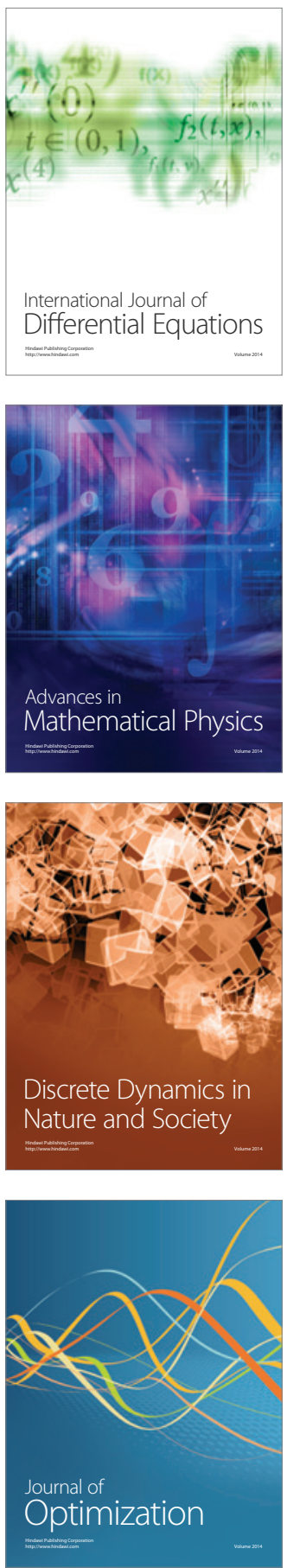